\title{
The slow demise of the long-lived SN 2005ip
}

\author{
Ori D. Fox ${ }^{\odot},{ }^{1 \star}$ Claes Fransson, ${ }^{2}$ Nathan Smith, ${ }^{3}$ Jennifer Andrews, ${ }^{3}$ K. Azalee Bostroem, ${ }^{4}$ \\ Thomas G. Brink, ${ }^{5}$ S. Bradley Cenko, ${ }^{6,7}$ Geoffrey C. Clayton ${ }^{\odot},{ }^{8}$ Alexei V. Filippenko, ${ }^{5,9} \ddagger$ \\ Wen-fai Fong, ${ }^{10}$ Joseph S. Gallagher ${ }^{11}$ Patrick L. Kelly, ${ }^{12}$ Charles D. Kilpatrick, ${ }^{13}$ Jon C. Mauerhan,,${ }^{5,14}$ \\ Adam M. Miller ${ }^{\oplus},{ }^{15,16} \dagger$ Edward Montiel, ${ }^{17}$ Maximilian D. Stritzinger, ${ }^{18}$ Tamás Szalai ${ }^{19,20}$ \\ and Schuyler D. Van Dyk ${ }^{21}$
}

Affiliations are listed at the end of the paper

Accepted 2020 July 28. Received 2020 July 10; in original form 2020 May 22

\begin{abstract}
The Type IIn supernova (SN IIn) 2005ip is one of the most well-studied and long-lasting examples of an SN interacting with its circumstellar environment. The optical light curve plateaued at a nearly constant level for more than five years, suggesting ongoing shock interaction with an extended and clumpy circumstellar medium (CSM). Here, we present continued observations of the SN from $\sim 1000$ to $5000 \mathrm{~d}$ post-explosion at all wavelengths, including X-ray, ultraviolet, near-infrared (NIR), and mid-infrared. The UV spectra probe the pre-explosion mass loss and show evidence for CNO processing. From the bolometric light curve, we find that the total radiated energy is in excess of $10^{50} \mathrm{erg}$, the progenitor star's pre-explosion mass-loss rate was $\gtrsim 1 \times 10^{-2} \mathrm{M}_{\odot} \mathrm{yr}^{-1}$, and the total mass lost shortly before explosion was $\gtrsim 1 \mathrm{M}_{\odot}$, though the mass lost could have been considerably larger depending on the efficiency for the conversion of kinetic energy to radiation. The ultraviolet through NIR spectrum is characterized by two high-density components, one with narrow high-ionization lines, and one with broader lowionization H I, He I, [O I], Mg II, and Fe II lines. The rich Fe II spectrum is strongly affected by Ly $\alpha$ fluorescence, consistent with spectral modelling. Both the Balmer and He I lines indicate a decreasing CSM density during the late interaction period. We find similarities to SN 1988Z, which shows a comparable change in spectrum at around the same time during its very slow decline. These results suggest that, at long last, the shock interaction in SN 2005ip may finally be on the decline.
\end{abstract}

Key words: circumstellar matter-dust, extinction-infrared: stars.

\section{INTRODUCTION}

Type IIn supernovae (SNe IIn; see Filippenko 1997 and Smith 2017 for reviews) are characterized by relatively narrow emission lines (Schlegel 1990) which are not associated with the SN explosion itself, but rather with dense circumstellar material (CSM) produced by preSN mass loss (Smith 2014). Shock interaction and dust formation in the dense CSM often result in significant emission ranging from $\mathrm{X}$-ray to radio wavelengths for many years post-explosion (e.g. Chevalier \& Fransson 2017; Fox et al. 2011, 2013).

SN 2005ip, discovered in NGC 2906 (Boles, Nakano \& Itagaki 2005) on 2005 November 5.163 (UT dates are used throughout this paper), is one of the more well-studied Type IIn explosions given its proximity to Earth $(\sim 35 \mathrm{Mpc})$ and the fact that it has remained detectable for nearly $15 \mathrm{yr}$ post-explosion. Fox et al. (2009) first reported a 3 yr near-infrared (NIR) light-curve plateau corresponding to newly formed dust in the cold, post-shock shell. Smith et al. (2009b) published an optical light curve that showed an initial linear (in mag $\mathrm{d}^{-1}$ ) decline from peak, followed by a late-time plateau

\footnotetext{
${ }^{\star}$ E-mail: ofox@stsci.edu

$\dagger$ Hubble Fellow.

$\ddagger$ Miller Senior Fellow.
}

attributed to ongoing shock interaction with the dense CSM. The late optical plateau matched the NIR plateau, and Smith et al. (2009b) presented spectra that revealed signatures of strong ongoing CSM interaction, as well as signatures of dust formation in both the SN ejecta and the post-shock cold dense shell. SN 2005ip was unusual in displaying very pronounced narrow coronal emission lines in its spectrum, indicating that clumpy CSM was being strongly irradiated by X-rays from the shock interaction (Smith et al. 2009b). Coronal lines have also been seen in the Type IIn SNe 1995N (Fransson et al. 2002), 2006jd (Stritzinger et al. 2012), and SN 2010jl (Fransson et al. 2014). Fox et al. (2010) obtained a Spitzer Space Telescope Infrared Spectrograph (Houck et al. 2004) spectrum of SN 2005ip [the only mid-IR (MIR) spectrum of a SN IIn to date], which revealed the presence of a cooler dust component associated with a pre-existing dust shell radiatively heated by this ongoing CSM interaction. Bevan et al. (2018) modelled the spectral line evolution and confirmed that a significant amount of dust can be explained by dust formation in the ejecta, and Nielsen, Hjorth \& Gall (2018) found the dust properties to be unlike those of Milky Way dust.

Stritzinger et al. (2012) continued to monitor SN 2005ip throughout $\sim 5$ yr post-explosion and showed that the optical and NIR light curves underwent little decline over that time. Katsuda et al. (2014) reported that X-ray observations at $\sim 6$ yr post-explosion exhibit a 
Table 1. HST imaging.

\begin{tabular}{|c|c|c|c|c|c|c|c|c|}
\hline $\begin{array}{l}\text { UT date } \\
\text { YYYMMDD }\end{array}$ & $\begin{array}{l}\text { Epoch } \\
\text { (d) }\end{array}$ & $\begin{array}{l}\text { Program } \\
\text { (GO) }\end{array}$ & PI & Instrument & Grating/Filter & $\begin{array}{c}\text { Central wavelength } \\
\text { (§) }\end{array}$ & $\begin{array}{l}\text { Exposure } \\
\text { (s) }\end{array}$ & Magnitude \\
\hline 20081118 & 1109 & 10877 & Weidong Li & WFPC2 & F450W & 4556.00 & 800 & $19.58(0.01)$ \\
\hline 20081118 & 1109 & 10877 & Weidong Li & WFPC2 & F555W & 5439.00 & 460 & $19.34(0.01)$ \\
\hline 20081118 & 1109 & 10877 & Weidong Li & WFPC2 & F814W & 8012.00 & 700 & $19.05(0.01)$ \\
\hline 20161029 & 4011 & 14668 & Filippenko & WFC3 & F336W & 3354.85 & 780 & $20.82(0.02)$ \\
\hline 20180111 & 4450 & 15166 & Filippenko & WFC3 & F814W & 8048.10 & 710 & $21.60(0.01)$ \\
\hline
\end{tabular}

Table 2. HST/STIS/MAMA spectroscopy.

\begin{tabular}{lcccc}
\hline $\begin{array}{l}\text { UT date } \\
\text { YYYYMMDD }\end{array}$ & $\begin{array}{c}\text { Epoch } \\
(\mathrm{d})\end{array}$ & $\begin{array}{c}\text { Program } \\
(\mathrm{GO})\end{array}$ & Grating & $\begin{array}{c}\text { Exposure } \\
(\mathrm{s})\end{array}$ \\
\hline 20140328 & 3065 & 13287 & G140L & $\begin{array}{l}4752 \\
\end{array}$ \\
20171021 & 4368 & \multirow{2}{*}{14598} & G230L & 2744 \\
& & & G140L & 17100 \\
& & & G230L & 19008 \\
\hline
\end{tabular}

significant decrease in flux compared to previous epochs, suggesting the forward shock had finally overtaken the dense CSM in which the SN exploded. Most recently, Smith et al. (2017) observed a temporary resurgence in the $\mathrm{H} \alpha$ luminosity, which they interpreted to be the result of the forward shock crashing into an additional dense shell located $\lesssim 0.05 \mathrm{pc}$ away, consistent with the distant pre-existing dust shell indicated by MIR observations (Fox et al. 2011). Overall, Smith et al. (2017) showed that the spectral evolution and X-ray emission from the decade-long CSM interaction phase of SN 2005ip was almost identical to the late interaction seen in SN 1988Z, the prototypical SN IIn with long-lasting CSM interaction.

The nature of the progenitors of both SN 2005ip and the broader Type IIn subclass remains ambiguous. The mass-loss rates of SNe IIn derived using various techniques are in the range $10^{-4}-10^{-1} \mathrm{M}_{\odot}$ $\mathrm{yr}^{-1}$ and the total CSM masses are several $\mathrm{M}_{\odot}$ (e.g. Smith et al. 2009b, 2007, 2008; Fox et al. 2009; Moriya et al. 2013; Ofek et al. 2014; Fransson et al. 2014; Katsuda et al. 2014; Smith 2017). Galactic analogues with such mass-loss rates and H-rich winds include luminous red supergiants, yellow hypergiants, and luminous blue variables (LBVs), each of which present further questions of their own (see Smith 2014 for a review). To complicate the interpretation even more, Habergham, James \& Anderson (2012) find that SNe IIn, including SN 2005ip, do not trace the most active star formation in galaxies, suggesting they are not exclusively associated with the most massive stars. Smith \& Tombleson (2015) note that extremely luminous LBVs themselves do not trace regions of recent massive star formation, and go on to explain this effect with a binary progenitor scenario. Nomoto, Iwamoto \& Suzuki (1995) also stressed the importance of binary evolution in various types of $\mathrm{SN}$ progenitors including SNe IIn. On the other hand, SNe IIn share many properties with SN impostors (Smith et al. 2011), and pre-existing dust shells are reminiscent of the impostors' pre-SN eruptions, such as SN 2009ip (e.g. Mauerhan et al. 2013), where the eruption was linked to an LBV progenitor (e.g. Smith et al. 2010). Taddia et al. (2015) find that long-lasting SNe IIn have similar host-galaxy metallicities as SN imposters, which may be produced by LBV outbursts and have traditionally been thought to arise from massive stars.

The pre-SN mass-loss history of SNe IIn may hold some clues since it probes the latest stages of massive-star evolution. Differences in wind speeds, densities, compositions, and asymmetries result in distinguishable observational behaviours. Given the dense CSM associated with most SNe IIn, X-rays from shock interaction are often absorbed, reprocessed, and re-emitted at UV (predominantly) and optical wavelengths, making these wavelengths optimal for tracing CSM interaction and, thereby, the progenitor's mass-loss history (e.g. Chevalier \& Fransson 1994; Chevalier \& Irwin 2011; Chevalier $\&$ Fransson 2017). Furthermore, when combined with other wavelengths, the UV may offer a quantitative estimate of the nucleosynthesis in the core of the star, which helps to constrain the initial mass of the progenitor prior to mass loss (Fransson et al. 2002, 2005, 2014).

Here, we present multiwavelength observations of SN 2005ip at very late epochs, including UV, optical, NIR, MIR, and radio. These data track the SN light curve as it declines in all bands. Following Stritzinger et al. (2012), throughout this paper we assume that the distance to NGC 2906 (the host galaxy) is $34.9 \mathrm{Mpc}$, and we adopt $E(B$ $-V)=0.047 \mathrm{mag}$ as the reddening (Smith et al. 2009b). When noted, we correct our spectral energy distributions (SEDs) and spectra for this colour excess assuming $R_{V}=A_{V} / E(B-V)=3.1$ using the reddening law of Cardelli, Clayton \& Mathis (1989). Section 2 presents the observations, while Section 3 analyses the light curve and spectral evolution. In Section 3.2.3, we take a closer look at the UV spectra. Finally, Section 4 provides a discussion and conclusion of the work.

\section{OBSERVATIONS}

\subsection{HST imaging}

Table 1 summarizes Hubble Space Telescope (HST) imaging of SN 2005ip. We obtained individual images from the Mikulski Archive for Space Telescopes, so they have been processed through the standard pipeline at the Space Telescope Science Institute (STScI). We obtained photometry from individual $f c$ frames using DOLPHOT (Dolphin 2016) with the following parameters: FitSky=3, RAper $=8$, and InterpPSFlib $=1$, and the TINYTIM model point spread functions (PSFs).

\subsection{HST/STIS}

SN 2005ip was observed twice with the HST Space Telescope Imaging Spectrograph (STIS) as part of programs GO-13287 and GO-14598 (PI: O. Fox), as summarized in Table 2. The 1D spectrum for each observation is extracted using the CALSTIS custom extraction software stistools.x1d. The default extraction parameters for STIS are defined for an isolated point source. For both G140L and G230L, the default extraction box width is 7 pixels and the background extraction box width is 5 pixels. 
Table 3. Spitzer photometry.

\begin{tabular}{lcccr}
\hline $\begin{array}{l}\text { JD - } \\
2,450,000\end{array}$ & $\begin{array}{c}\text { Epoch } \\
(\mathrm{d})\end{array}$ & PID & $\begin{array}{l}3.6 \mu \mathrm{m} \\
\left(10^{17} \mathrm{erg} \mathrm{s}^{-1}\right.\end{array}$ & $\begin{array}{c}4.5 \mu \mathrm{m} \\
\left.\mathrm{cm}^{-2} \AA^{-1}\right)\end{array}$ \\
\hline 4628 & 948 & 50256 & $13.62(0.31)$ & $10.96(0.21)$ \\
5737 & 2057 & 80023 & $6.44(0.20)$ & $5.87(0.15)$ \\
6476 & 2796 & 90174 & $3.36(0.15)$ & $3.02(0.11)$ \\
6845 & 3165 & 10139 & $2.68(0.14)$ & $2.32(0.09)$ \\
7229 & 3549 & 11053 & $2.22(0.13)$ & $1.80(0.08)$ \\
8347 & 4667 & 14098 & $1.56(0.11)$ & $1.05(0.07)$ \\
\hline
\end{tabular}

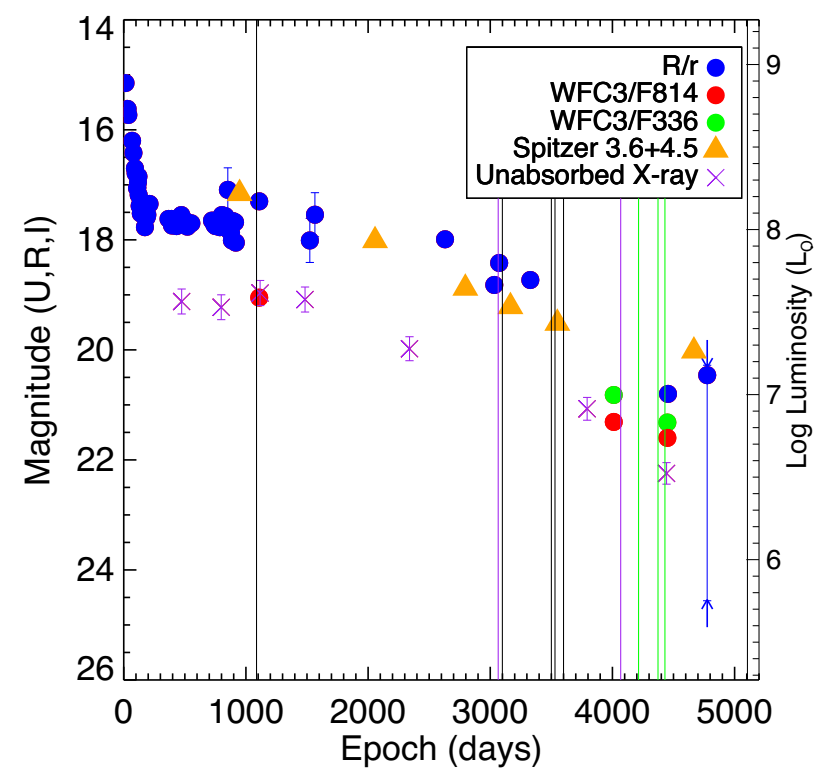

Figure 1. Multiwavelength photometry of SN 2005ip, including data presented in this paper (Smith et al. 2009b; Fox et al. 2010; Stritzinger et al. 2012; Katsuda et al. 2014; Szalai et al. 2019). Vertical identify epochs on which the spectra presented here were obtained (black=Keck, green=MMT, and purple=Chandra). Note that the left-hand ordinate axis applies only to the $R / r$ band, F814, and F336 photometry, while the right-hand ordinate axis applies to the Spitzer integrated and X-ray luminosities. The most complicated point in Fig. 1 is the $2018 R$-band photometry from Keck (day 4776), when the $\mathrm{SN}$ is quite faint and comparable in broad-band flux to the underlying $\mathrm{H}$ II region. Aperture photometry, which includes some of the underlying H II region, yields a magnitude of 19.92 , which we take to be the SN upper limit (Table 4). We also obtained a final epoch of Keck $R$-band imaging in 2019. Under the assumption that the SN had faded completely (although it likely had not), we can use the 2019 data as a template for subtraction from the 2018 data. This yields a magnitude of 24.54 , which we take to be the SN lower limit in 2018. In reality, the actual SN flux on day 4776 is somewhere between these limits.

\subsection{Warm Spitzer/IRAC photometry}

The Warm Spitzer Infrared Array Camera (IRAC, Fazio et al. 2004) obtained several epochs of data for SN 2005ip, summarized in Table 3 and plotted in Fig. 1. We downloaded co-added and calibrated Post Basic Calibrated Data ( $\mathrm{pbcd}$ ) from the Spitzer Heritage Archive. Standard aperture photometry was performed with a radius defined by a fixed multiple of the PSF full width at half-maximum intensity (FWHM). Template subtraction is typically implemented to remove contributions from the underlying galaxy, but in this case, no Spitzer template exists. Furthermore, due to the rapid flux variations of the underlying galaxy, a standard annulus does not allow for selection of pixels corresponding to a local background associated with the SN (e.g. Fox et al. 2011; Szalai et al. 2019). Instead, we selected our own background region. The standard deviation of the background variations is smaller than the measured noise in the aperture photometry, even at the latest epochs, and does not contribute substantially to our error bars. Most of these data were recently published by Szalai et al. (2019), but day 4667 is newly published here. The photometry for epochs 948 and 2057 does not precisely match the photometry of Fox et al. $(2010,2011,2013)$ because the analysis in those papers implemented slightly different routines consisting of either different sized apertures or PSF fitting photometry, but they are within the error bars. All Spitzer photometry in this paper was calculated using aperture photometry with consistent aperture sizes.

For plotting purposes in Fig. 1, we derive the integrated Spitzer luminosity by fitting a simple dust-mass model to the two MIR data fluxes, similar to those described by Fox et al. (2011). In this case, we assume $0.1 \mu \mathrm{m}$ graphite grains given the lack of the $\sim 10 \mu \mathrm{m}$ silicate feature in the MIR spectrum (Fox et al. 2010; Williams \& Fox 2015).

\subsection{Optical and NIR photometry}

Tables 4 and 5 list and Fig. 1 plots the new optical and NIR photometry of SN 2005ip. We include some data obtained with the Reionization And Transients InfraRed camera (RATIR; Butler et al. 2012; Fox et al. 2012) mounted on the $1.5 \mathrm{~m}$ Johnson telescope at the Mexican Observatorio Astronomico Nacional on Sierra San Pedro Mártir in Baja California, México (Watson et al. 2012). The data were reduced, co-added, and analysed using standard CCD and IR processing and aperture photometry techniques, utilizing online astrometry programs SEXTRACTOR and SWARP. ${ }^{1}$

We also present two epochs of $r$ and $i$ photometry obtained during 2014 at Las Campanas Observatory with the $2.5 \mathrm{~m}$ du Pont telescope by the Carnegie Supernova Project (CSP; Hamuy et al. 2006). These images were reduced following standard procedures. PSF photometry of the SN was computed in the natural system using the local sequence stars presented by Stritzinger et al. (2012). The reported photometric uncertainties account for both instrumental and nightly zero-point errors.

Additional NIR photometry was obtained from the $3.8 \mathrm{~m}$ United Kingdom Infrared Telescope (UKIRT) on Maunakea using WFCAM2. JHK observations were pipeline reduced by the Cambridge Astronomical Survey Unit. Aperture photometry was performed using the DAOPHOT package in IRAF. ${ }^{2}$ Uncertainties were calculated by adding in quadrature photon statistics and zero-point deviation of the standard stars for each epoch.

One epoch of $g^{\prime}$ and $r^{\prime}$ photometry was obtained with the MultiObject Double Spectrograph (Byard \& O'Brien 2000) on the Large Binocular Telescope (LBT) on 2018 January 16. The $3 \times 240 \mathrm{~s}$ images in each filter were reduced and stacked using standard IRAF procedures, and zero-points were calculated using Sloan Digital Sky Survey (SDSS) standard stars in the field. Uncertainties were calculated in the same manner as for the UKIRT data.

All magnitudes were initially calculated in their respective telescopes natural system. Although not every telescope has a published report detailing their system, they all follow similar techniques as

\footnotetext{
${ }^{1}$ SEXTRACTOR and SWARP can be accessed from http://www.astromatic.net/s oftware.

${ }^{2}$ IRAF: the Image Reduction and Analysis Facility is distributed by the National Optical Astronomy Observatory, which is operated by the Association of Universities for Research in Astronomy (AURA), Inc., under cooperative agreement with the US National Science Foundation (NSF).
} 
Table 4. Ground-based optical photometry.

\begin{tabular}{|c|c|c|c|c|c|c|c|c|c|}
\hline $\begin{array}{l}\text { JD - } \\
2,450,000\end{array}$ & $\begin{array}{l}\text { Epoch } \\
\text { (d) }\end{array}$ & $g^{\prime}$ & $B$ & $V$ & mag $R$ & $r$ & $I$ & $i$ & Instrument \\
\hline 6310 & 2630 & - & - & - & - & $17.99(0.1)$ & - & $17.95(0.04)$ & RATIR \\
\hline 6713 & 3033 & - & - & - & - & $18.82(0.1)$ & - & $18.76(0.01)$ & CSP \\
\hline 6753 & 3073 & - & - & - & - & $18.42(0.1)$ & - & $18.31(0.06)$ & RATIR \\
\hline 7008 & 3328 & - & - & - & - & $18.73(0.1)$ & - & $18.76(0.01)$ & CSP \\
\hline 8134 & 4455 & $21.54(0.1)$ & - & - & - & $20.80(0.1)$ & - & - & LBT \\
\hline 8455 & 4776 & - & $>20.83$ & $>20.88$ & $19.92-24.54$ & - & $>19.68$ & - & Keck/LRIS \\
\hline
\end{tabular}

Table 5. Ground-based NIR photometry.

\begin{tabular}{lccccccc}
\hline JD - & Epoch & $Z$ & $Y$ & $J$ & $H$ & $K$ & Instrument \\
$2,450,000$ & $(\mathrm{~d})$ & & & \multicolumn{2}{l}{ mag } \\
\hline 6310 & 2630 & $17.47(0.01)$ & - & $16.27(0.01)$ & $15.65(0.02)$ & - & RATIR \\
6753 & 3073 & $17.91(0.01)$ & - & $16.77(0.02)$ & $16.09(0.02)$ & - & RATIR \\
7435 & 3755 & - & - & $19.81(0.1)$ & $18.91(0.1)$ & $17.3(0.1)$ & UKIRT \\
7451 & 3771 & - & - & - & - & $17.2(0.1)$ & UKIRT \\
7483 & 3803 & - & - & - & $18.96(0.1)$ & $17.2(0.1)$ & UKIRT \\
\hline
\end{tabular}

Table 6. Ground-based spectroscopy.

\begin{tabular}{lcccc}
\hline $\begin{array}{l}\text { JD }- \\
2,450,000\end{array}$ & $\begin{array}{c}\text { Epoch } \\
(\mathrm{d})\end{array}$ & Instrument & $\begin{array}{c}\text { Res. } \\
(\AA)\end{array}$ & $\begin{array}{c}\text { Exp. } \\
(\mathrm{s})\end{array}$ \\
\hline 4584 & 905 & Keck/LRIS & $\sim 9$ & 1200 \\
6246 & 2567 & Keck/DEIMOS & $\sim 3$ & 2400 \\
6778 & 3099 & Keck/LRIS & $\sim 6$ & 1200 \\
7372 & 3693 & Keck/DEIMOS & $\sim 3$ & 2400 \\
7449 & 3770 & Keck/DEIMOS & $\sim 3$ & 2400 \\
7893 & 4214 & MMT/BC & $\sim 1$ & 1200 \\
8052 & 4373 & MMT/BC & $\sim 1$ & 1200 \\
8109 & 4430 & MMT/BC & $\sim 1$ & 1200 \\
8784 & 5105 & Keck/LRIS & $\sim 6$ & 1200 \\
\hline
\end{tabular}

CSP (Contreras et al. 2010). All photometric calibration was then performed using field stars with reported fluxes in both Two Micron All Sky Survey (2MASS, Skrutskie et al. 2006) and the SDSS Data Release 9 Catalogue (Ahn et al. 2012). Uncertainties are dominated by errors associated with catalogue stars.

The most complicated point in Fig. 1 is the $2018 R$-band photometry from Keck (day 4776), when the $\mathrm{SN}$ is quite faint and comparable in broad-band flux to the underlying $\mathrm{H}$ II region. Aperture photometry, which includes some of the underlying $\mathrm{H}$ II region, yields a magnitude of 19.92, which we take to be the SN upper limit (Table 4). We also obtained a final epoch of Keck $R$-band imaging in 2019. Under the assumption that the SN had faded completely (although it likely had not), we can use the 2019 data as a template for subtraction from the 2018 data. This yields a magnitude of 24.54 , which we take to be the SN lower limit in 2018. In reality, the actual SN flux on day 4776 is somewhere between these limits.

\subsection{Optical spectroscopy}

Table 6 lists and Fig. 2 plots the new optical spectra of SN 2005ip. We obtained some spectra with the Low Resolution Imaging Spectrometer (LRIS; Oke et al. 1995) mounted on the $10 \mathrm{~m}$ Keck I telescope and the DEep Imaging Multi-Object Spectrograph (DEIMOS; Faber et al. 2003) mounted on the $10 \mathrm{~m}$ Keck II telescope. For the Keck/LRIS spectra, we observed with a 1 arcsec wide slit and used either the $600 / 4000$ or $400 / 3400$ grisms on the blue side and the $400 / 8500$ grating on the red side. This observing setup resulted in wavelength coverage from 3200 to $9200 \AA$ and a typical resolution of 5-7 $\AA$. For the Keck/DEIMOS spectra, we observed with a 1 arcsec wide slit and the $1200 / 7500$ grating. This observing setup resulted in wavelength coverage from $4750-7400 \AA$ and a typical resolution of $\sim 3 \AA$. In both cases, we aligned the slit the parallactic angle to minimize differential light losses (Filippenko 1982). These spectra were reduced using standard techniques (e.g. Foley et al. 2003; Silverman et al. 2012), Routine CCD processing and spectrum extraction were implemented using the optimal algorithm of Horne (1986). We flux calibrated these spectra and removed telluric absorption lines using algorithms defined by Wade \& Horne (1988) and Matheson et al. (2000).

We also obtained three epochs of spectroscopy with the Bluechannel (BC) spectrograph on the $6.5 \mathrm{~m}$ Multiple Mirror Telescope (MMT) using the $12001 \mathrm{~mm}^{-1}$ grating centred at $6300 \AA$ (see Smith et al. 2017). We performed standard reductions, including bias subtraction, flat-fielding, and optimal spectral extraction. We flux calibrated these spectra using spectrophotometric standards observed at similar airmasses.

\subsection{Chandra X-ray photometery}

The Chandra X-ray Observatory (CXO) Advanced CCD Imaging Spectrometer (ACIS; Garmire et al. 2003) observed SN 2005ip, summarized in Table 7 and plotted in Fig. 1. As a reference, we also include details of the previous epoch of $C X O$ observations from 2016 (Smith et al. 2017). Similar to Smith et al. (2017), we performed photometry and spectral extraction using the specextract package within the HEASOFT ${ }^{3}$ CIAO software suite (Blackburn 1995). We model source and background spectra simultaneously using the SHERPA package. For the source, we assume an absorbed singletemperature thermal plasma model (APEC) having solar abundances as defined by Asplund et al. (2009). For the background, we assume a simple power law. We set the equivalent neutral hydrogen column density in the interstellar medium (ISM) of $N_{\mathrm{H}}(\mathrm{ISM})=$ $3.7 \times 10^{20} \mathrm{~cm}^{-2}$ (Katsuda et al. 2014). We also allowed for an additional intrinsic source of absorption for the SN. The fits rely on a

\footnotetext{
${ }^{3} \mathrm{http} / / /$ heasarc.gsfc.nasa.gov/ftools
} 


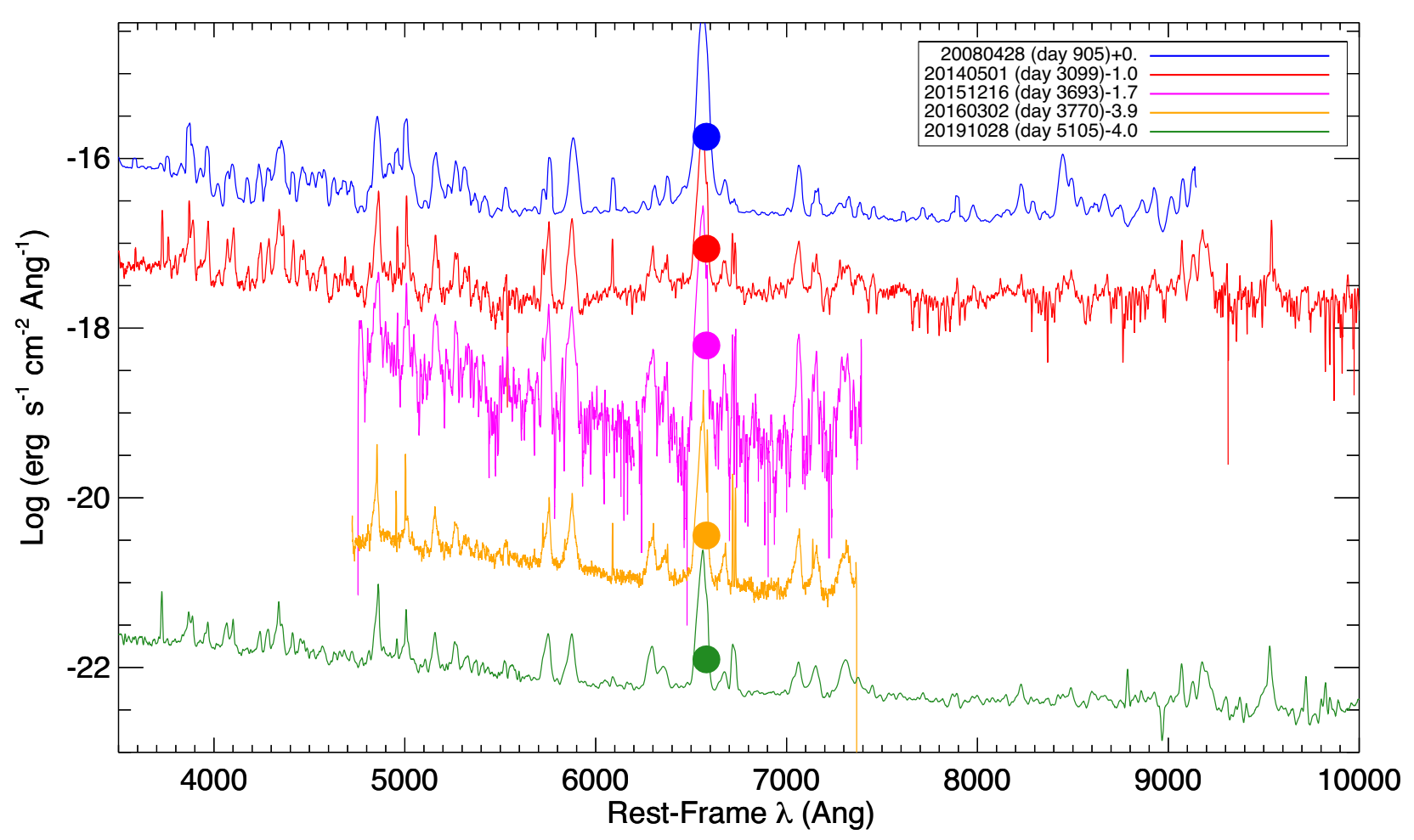

Figure 2. Some of the ground-based optical spectra summarized in Table 6. The spectra are scaled to the optical photometry for absolute comparisons at each epoch.

Table 7. Chandra observations of SN 2005ip.

\begin{tabular}{|c|c|c|c|c|c|c|c|c|}
\hline Instrument & $\begin{array}{c}\text { JD - } \\
2,450,000\end{array}$ & $\begin{array}{c}\text { Days after } \\
\text { outburst }\end{array}$ & $\begin{array}{l}\text { Exposure } \\
\text { (ks) }\end{array}$ & $\begin{array}{l}\text { Counts } \\
\left(10^{-3} \text { counts s }{ }^{-1}\right)\end{array}$ & $\begin{array}{c}N_{\mathrm{H}} \\
\left(10^{22} \mathrm{~cm}^{-2}\right)\end{array}$ & $\begin{array}{c}k T \\
(\mathrm{keV})\end{array}$ & $\begin{array}{c}0.5-8 \mathrm{keV} \\
\text { Unabsorbed Flux } \\
\left(10^{-13} \mathrm{erg} \mathrm{s}^{-1} \mathrm{~cm}^{-2}\right)\end{array}$ & $\begin{array}{l}\text { Luminosity } \\
\left(10^{40} \mathrm{erg} \mathrm{s}^{-1}\right)\end{array}$ \\
\hline ACIS-S & 8122 & 4453 & 41.22 & 619 & $0.11_{-0.11}^{+0.25}$ & $3.2_{-1.2}^{+2.0}$ & $1.2_{-1.1}^{+1.0}$ & 1.3 \\
\hline
\end{tabular}

$\chi^{2}$ statistic with a Gehrels variance function. In this case, we obtained a reduced $\chi^{2}$ value of 35 for 64 degrees of freedom. We use these fits to derive photon energies in the range $0.5-8.0 \mathrm{keV}$. Compared to our previously reported Chandra/ACIS observation on 2016 Apr. 3 (Smith et al. 2017), SN 2005ip exhibits a factor of $\sim 2$ reduction in the intrinsic flux. The temperature and self-absorption parameters of our thermal plasma model, however, have not changed significantly.

\section{ANALYSIS}

\subsection{Light-curve evolution, bolometric luminosity, and radiated energy}

Fig. 1 shows decreasing fluxes at all wavelengths at days $\gtrsim 1500$ postexplosion, which is consistent with other SNe IIn observed at such late epochs (Fox et al. 2011). Based on the X-ray observations alone, Katsuda et al. (2014) attribute the decreasing flux to the forward shock having finally overtaken the dense CSM in which the SN exploded. Fig. 2 shows that most of the decreasing flux occurs in the strength of $\mathrm{H} \alpha$ line, although the later spectra suggest that there may be some additional contribution from outside the $\mathrm{H} \alpha$ line, possibly from a faint reflected light echo or a blend of very faint CSM interaction lines and their wings.
We use the data from Fig. 1 to construct a quasi-bolometric luminosity light curve (Fig. 3), which we will refer to simply as the bolometric light curve hereafter. For the optical, we use our $r$ band photometry and scale magnitudes to correspond to the optical luminosities from Stritzinger et al. (2012) in the range 350-900 d, assuming that the spectrum does not change appreciably after this epoch. This includes both the 'hot' component and the lines in Stritzinger et al. (2012), giving

$L_{\text {optical }}=10^{-0.4 r+48.45}$.

The IR luminosities are described above. The MIR luminosities do not include the cold component discussed by Fox et al. (2010), whose origin may be from more distant gas. Taken all together, the bolometric luminosity may be underestimated by at most 50 per cent. Because we do not include the far-IR, UV, or X-rays, this is likely to be a lower limit to the luminosity. The observed X-rays give an additional contribution of ( 15 per cent-20 per cent). Unfortunately, this component is not known before $460 \mathrm{~d}$. For the total bolometric output only the observed X-ray luminosity should be included, not the fraction absorbed by the CSM, which is thermalized into UV and optical radiation. This fraction is most likely increasing for the earlier epochs, approaching 100 per cent as the column density of the CSM and ejecta ahead of the shock increases at early epochs. 


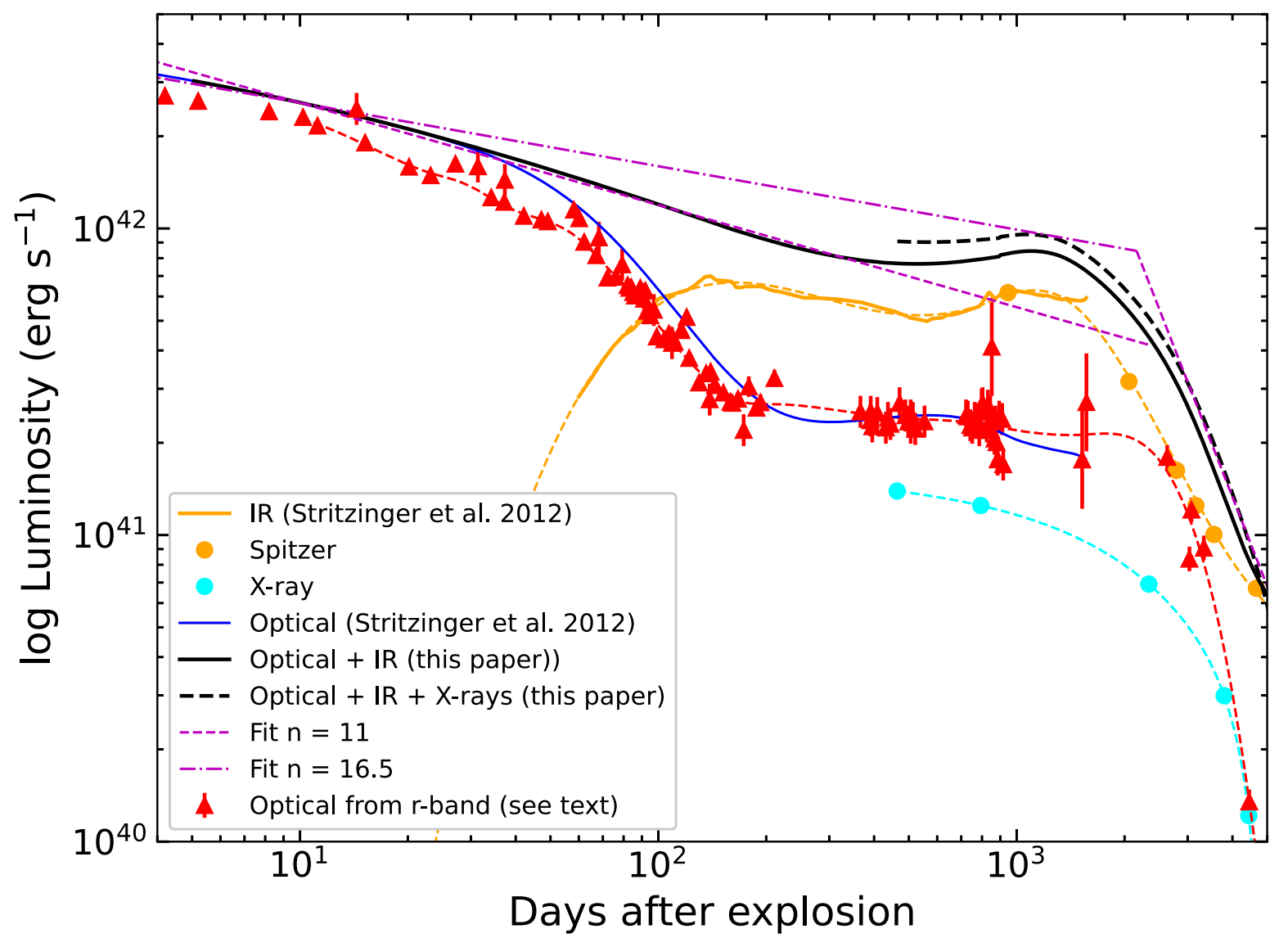

Figure 3. Quasi-bolometric light curve of SN 2005ip. The solid lines are the optical (blue) and IR (orange) light curves from Stritzinger et al. (2012), while the dashed lines are fits to the $r$-band (red) and Spitzer (orange) light curves, with photometry from this paper. The solid black line is the total constructed by merging the light curves from Stritzinger et al. (2012) and this paper. The dashed magenta lines show power-law fits to the two phases of the total luminosity (including and excluding X-rays, which are shown in cyan).

This is highly model dependent, and we do therefore not attempt to model it. To estimate the effect, however, we add the observed X-ray luminosity to the optical and IR contributions, shown as the dashed black line in Fig. 3.

Fig. 3 shows that the optical plus IR luminosity up to $\sim 700 \mathrm{~d}$ can be well described by a power law in time, $L \approx 2.6 \times$ $10^{42}(t /(10 \mathrm{~d}))^{-0.33} \mathrm{erg} \mathrm{s}^{-1}$. The power-law decay during the first phase can be well described by the similarity solution for a radiative shock in a CSM with a steady mass-loss rate (e.g. Chevalier \& Fransson 2017): $L \propto v_{\mathrm{s}}^{3} \propto t^{-3 /(n-2)}$, where $n$ is the power-law index of the ejecta density profile. If we use only the total optical plus IR light curve, the luminosity decrease corresponds to $n \approx 11$, somewhat steeper than found for SN 2010j1 (Fransson et al. 2014).

When we also include the X-rays, the dip in the optical plus IR light curve before the plateau partly fills in, increasing the luminosity by $\sim 20$ per cent. If we fit the luminosity from $10 \mathrm{~d}$ to the peak of the 'bump' at $\sim 1000 \mathrm{~d}$, we find $L \approx 2.6 \times 10^{42}(t /(10 \mathrm{~d}))^{-0.21} \mathrm{erg} \mathrm{s}^{-1}$, corresponding to $n=16.5$. Note, however, that this excludes any $\mathrm{X}$-ray contribution at earlier epochs which would steepen the decline and decrease $n$. In this context, we also note that a short-lived eruption, as is probably the case here, may have a density profile different from a steady $\rho \propto r^{-2}$ wind.

After $\sim 2000 \mathrm{~d}$ the light curve breaks, and the decay is steeper, with $L \propto(t /(10 \mathrm{~d}))^{-3}$. This behaviour signals the breakout of the shock wave from at least part of the dense CSM. Compared again to SN $2010 \mathrm{j} 1$, where the break occurred at $\sim 350 \mathrm{~d}$, this is considerably later.
We integrate the bolometric light curve to estimate a total radiated energy of $1.7 \times 10^{50} \mathrm{erg}$. We add $\sim 1 \times 10^{49} \mathrm{erg}$ from the X-rays. As we discuss above, however, this is most likely only a lower limit to the total radiated energy.

Using the bolometric light curve we can also estimate the massloss rate. Assuming a radiative shock in a steady wind, the total luminosity is given by

$L=\frac{1}{2} \epsilon \frac{\dot{M}}{v_{\mathrm{w}}} v_{\text {shock }}^{3}$,

where $\epsilon \leq 1$ is the efficiency for conversion, $v_{\text {shock }}$ is the shock velocity, $\dot{M}$ is the mass-loss rate, and $v_{\text {w }}$ is the wind velocity. From the narrow high-ionization lines we estimate the velocity of the preshocked CSM to be $\sim 100 \mathrm{~km} \mathrm{~s}^{-1}$. If we write the luminosity as $L(t)=$ $L\left(t_{*}\right)\left(t / t_{*}\right)^{\alpha}$ and $v_{\text {shock }}=v_{\text {shock }}\left(t_{0} / t\right)^{1 /(n-2)}$, we get

$\dot{M}=\frac{2 L\left(t_{*}\right) v_{\mathrm{w}}}{\epsilon v_{\text {shock }}\left(t_{0}\right)^{3}}\left(\frac{t_{*}}{t_{0}}\right)^{3 /(n-2)}$.

The shock velocity injects the largest uncertainty in the above equation. Because of electron scattering at early epochs, one cannot use the maximum wavelength shift of the blue wing. Fig. 6 of Smith et al. (2009b) shows that this line has a 'shoulder' at $\sim 5300 \mathrm{~km} \mathrm{~s}^{-1}$ on the blue side in the spectra at $\sim 100 \mathrm{~d}$. As discussed in detail by Taddia et al. (2020), this shoulder may be caused by the macroscopic shock velocity, in contrast to the wings caused by electron scattering. Our late-time $\mathrm{H} \alpha$ spectra (Section 3.2.4) indicate a velocity 
of $\sim 2700 \mathrm{~km} \mathrm{~s}^{-1}$ at $\sim 1000 \mathrm{~d}$, but the line profile still suggests contributions from electron scattering. Given this uncertainty, we will therefore scale the mass loss to a velocity of $3000 \mathrm{~km} \mathrm{~s}^{-1}$ at $1000 \mathrm{~d}$, as in equation (4).

With $t_{*}=10 \mathrm{~d}, L\left(t_{*}\right)=2.6 \times 10^{42} \mathrm{erg} \mathrm{s}^{-1}$, and $t_{0}=1000 \mathrm{~d}$, we get

$\dot{M}=\frac{6.6 \times 10^{-3}}{\epsilon}\left[\frac{v_{\mathrm{w}}}{100 \mathrm{~km} \mathrm{~s}^{-1}}\right]\left[\frac{v_{\text {shock }}(1000 \mathrm{~d})}{3000 \mathrm{~km} \mathrm{~s}^{-1}}\right]^{-3} \mathrm{M}_{\odot} \mathrm{yr}^{-1}$.

The total mass of the SN 2005ip gas shell up to the break at $t_{\text {break }}$ is then

$\mathrm{M}_{\mathrm{tot}}=\frac{\dot{M}}{v_{\mathrm{w}}} v_{\text {shock }}\left(t_{0}\right) t_{\text {break }}\left(\frac{t_{0}}{t_{\text {break }}}\right)^{1 /(n-2)}$.

If we assume that the shock has exited the densest part of the CSM at $t_{\text {break }} \approx 2000 \mathrm{~d}$ and with $\dot{M}$ from equation (4), we get

$\mathrm{M}_{\mathrm{tot}}=\frac{1.0}{\epsilon}\left[\frac{v_{\text {shock }}(1000 \mathrm{~d})}{3000 \mathrm{~km} \mathrm{~s}^{-1}}\right]^{-2}\left(\frac{t_{\text {break }}}{2000 \mathrm{~d}}\right)^{8 / 9} \mathrm{M}_{\odot}$.

Equations (4) and (6) together give a timescale of $\sim 150\left(v_{\text {shock }}(1000 \mathrm{~d}) / 3000 \mathrm{~km} \mathrm{~s}^{-1}\right)\left(v_{\mathrm{w}} / 100 \mathrm{~km} \mathrm{~s}^{-1}\right)^{-1} \mathrm{yr}$ for the strong mass-loss episode.

If we instead use the flatter evolution of the luminosity in Fig. 3 with $n=16.5$, the coefficient in equation (4) becomes $1.2 \times 10^{-2} \mathrm{M}_{\odot} \mathrm{yr}^{-1}$, and in equation (6) the coefficient becomes $1.85 M_{\odot}$.

As seen from equations (4) and (6), besides the value of $v_{\text {shock, an }}$ important uncertainty in these estimates is the efficiency parameter, $\epsilon$, which depends on the importance of shock-wave instabilities and other multidimensional effects (Taddia et al. 2020), and may be in the range $\sim 0.1-1$. For these reasons, the total radiated energy and total mass should be taken as lower limits. In addition, we only integrated the total mass swept to the break at $\sim 2000 \mathrm{~d}$. Even if the light-curve steepening is caused by a decreasing density, the later evolution will certainly contribute to a substantial additional mass.

Other studies using X-ray and MIR data favour total CSM masses even higher than $10 \mathrm{M}_{\odot}$ (Stritzinger et al. 2012; Katsuda et al. 2014). These results are consistent with mass-loss rates that are all nearly $10^{-2} \mathrm{M}_{\odot} \mathrm{yr}^{-1}$ for a period of several hundred years leading up to the progenitor's explosion, similar to what we find above. The superluminous Type IIn SN 2010j1, for comparison, had a mass-loss rate of nearly $10^{-2} \mathrm{M}_{\odot} \mathrm{yr}^{-1}$ and total mass loss of $\gtrsim 3 \mathrm{M}_{\odot}$.

\subsection{Spectral modelling and line identifications}

Basic modelling can be used to infer some qualitative estimates of the physical conditions in the CSM and SN, although this should not be confused with a fully self-consistent spectral modelling (e.g. Dessart, Audit \& Hillier 2015). Already, there has been extensive discussion of the rich line spectra of SN 2005ip (Smith et al. 2009b; Smith et al. 2017; Stritzinger et al. 2012). The H I, He I, [N II], [O I], Mg II, and [Ca II] lines have FWHM $\approx 1500 \mathrm{~km} \mathrm{~s}^{-1}$ and are understood to originate from a denser, optically thick medium. We will refer to these as the low-ionization component. In contrast, the high-ionization lines are all narrow, originating in the pre-shocked CSM with FWHM $\approx 350 \mathrm{~km} \mathrm{~s}^{-1}$.

\subsubsection{Low-ionization lines and Lyd fluorescence}

As a tool for line identifications and for the diagnostics we have calculated a synthetic spectrum, including H I, He I, N II, O I, Mg II,
CaII, and Fe II, which account for most of the spectral features. For Hi, He I, N II, O I, and Fe VII, we use multilevel model atoms, including collisional and radiative processes. We assume a two-zone model with temperature and density as parameters: one zone for the neutral and singly ionized elements and one zone for the highionization ions. We include optical-depth effects for the lines in the Sobolev approximation. The ionic abundances are treated as parameters. The atomic data for high-ionization stages are from the CHIANTI data base (Landi et al. 2012). For H I, we use collision rates from Anderson et al. (2002) and for He I from Benjamin, Skillman \& Smits (1999). Radiative transition rates and energy levels are mainly from NIST.

We have not attempted a similar calculation for Fe II, in spite of the large number of lines in the spectrum. This requires a more detailed calculation, including radiative excitation by overlapping lines, leading to fluorescence through line coincidences. While this is not a problem for the ions above, there are strong indications that the Fe II spectrum is much affected by fluorescence. In particular, the prominent features at $\sim 8450-9200 \AA$, not usually seen in SN spectra, are noteworthy. The peaks near $9200 \AA$ do not coincide with any Paschen lines or $\mathrm{Mg}$ II, both of which are detected at longer wavelengths. Instead, we argue that this emission arises from Fe II lines powered by fluorescence.

Excitation of $\mathrm{Fe}$ II by fluorescence from Ly $\alpha$ was first discussed for cool and symbiotic stars (Johansson \& Jordan 1984), and later for active galactic nuclei (AGNs) and LBVs, in particular for $\eta$ Carinae (see e.g. Johansson \& Hamann 1993; Hartman 2013, for reviews). In the SN context fluorescence by Ly $\alpha$ was found to be important for the Type IIn SN 1995N (Fransson et al. 2002). The most important branch is pumping by $\operatorname{Ly} \alpha$, primarily from the $a^{4} D^{e}$ excited level at $1.04 \mathrm{eV}$ in Fe II to levels $\sim 11.2 \mathrm{eV}$ above the ground state (Sigut \& Pradhan 1998, 2003). The cascade from these levels results in NIR lines at $\sim 8450-9200 \AA$ and UV lines at $\sim 2300-2900 \AA$. While both the UV lines and optically forbidden lines may also be excited by thermal collisions, the NIR lines require a very large excitation energy and are characteristic signatures of $\operatorname{Ly} \alpha$ pumping.

In SN 2005ip, the strong Ly $\alpha$ line reaches to $\sim 15 \AA$ on the red side, and more on the blue, although the blue is contaminated by the geocoronal Ly $\alpha$ and interstellar absorption (Fig. 6). Sigut \& Pradhan (2003) find 15 transitions from the $a^{4} D^{e}$ level within $\pm 3 \AA$ of Ly $\alpha$, so pumping can occur in a large number of transitions. Pumping from other low levels of Fe II may occur.

To model the Fe II spectrum we have therefore taken two approaches. In one, we have used the relative intensities by Sigut $\&$ Pradhan (2003), based on theoretical calculations. These are tuned for typical AGN conditions and may therefore give somewhat different intensities from those expected in the CSM of an SN. In particular, the velocity field is very different and non-local scattering may be important, resulting in other pumping channels. The qualitative results should, however, be similar. In the other approach, we use the observed UV to NIR spectrum of $\eta$ Carinae by Zethson et al. (2012). The line intensities are convolved with Gaussian profiles and we add a continuum with $F_{\lambda} \propto \lambda$.

Comparing the spectra in Fig. 2 we see little evolution between day 905 and at least to day 3770 . Because the spectrum around $3100 \mathrm{~d}$ has a coverage in both the UV and the NIR we will here concentrate on this spectrum. We return to the other spectra and changes between these below.

Fig. 4 shows the result of a 'best-fitting' calculation for the two $\mathrm{Fe}$ II line cases mentioned above. For both models, we note a steep Balmer decrement, with $F(\mathrm{H} \alpha) / F(\mathrm{H} \beta) \approx 9$. This ratio is comparable to that of other SNe IIn (Fransson et al. 2014) and is mainly a result of 


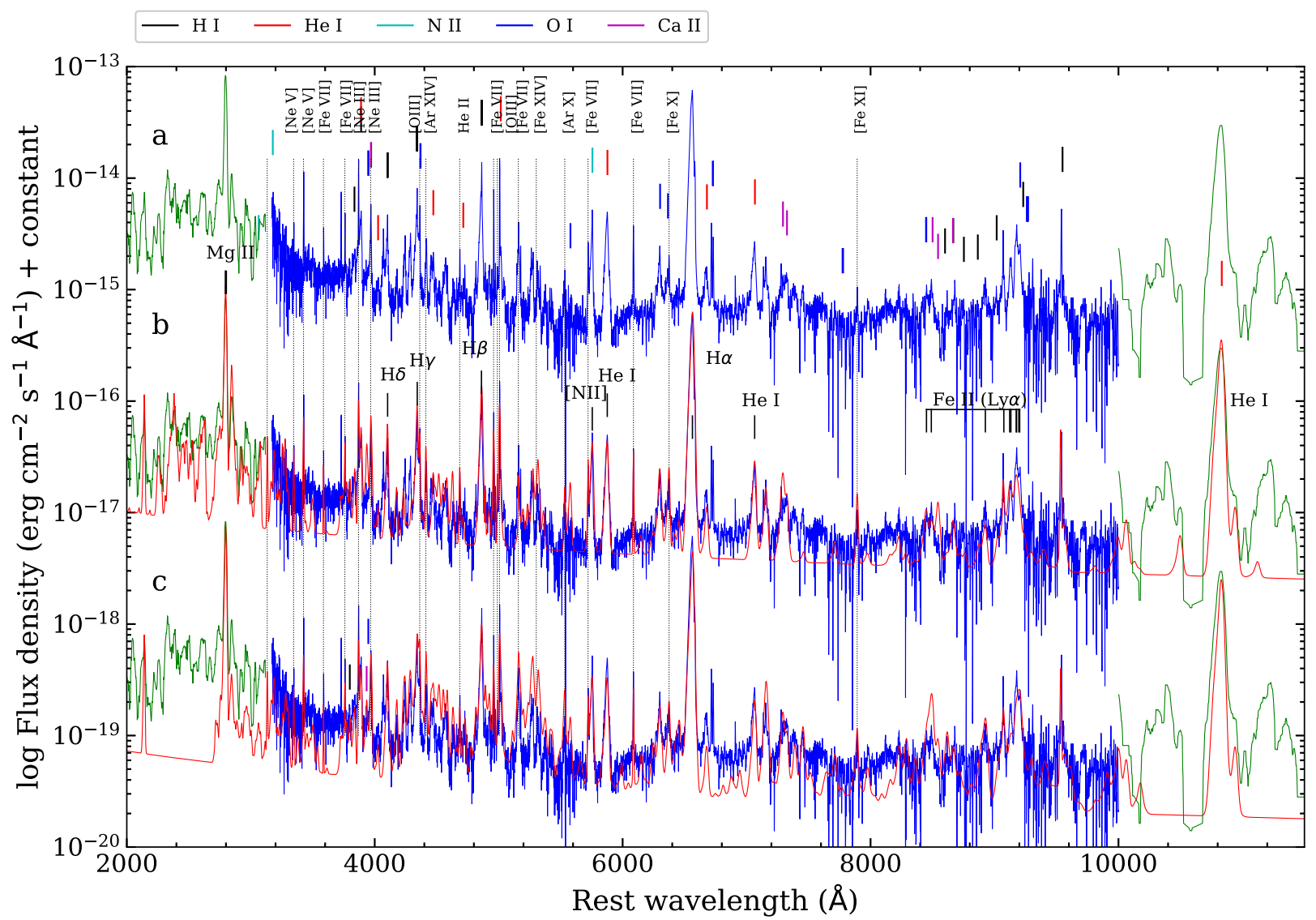

Figure 4. Synthetic spectra with line identifications for the UV to MIR spectrum of SN 2005ip at 3065-3165 d. The upper spectrum, (a), shows the observed data (green $=$ near-UV and NIR, blue = optical) with colour-coded line identifications given in the legend above the figure, plus a number of marked high-ionization lines, described in the text. The middle spectrum, (b), shows the same observed data together with the synthetic spectrum (red). The Fe II spectrum used is taken from the calculations by Sigut \& Pradhan (2003). Spectrum (c) is the same, but with the the observed Fe II spectrum of $\eta$ Carinae from Zethson et al. (2012). Note especially the strong NIR Fe II lines at $\sim 8450-9200 \AA$ A , powered by fluorescence from Ly $\alpha$.

the large optical depth of $\mathrm{H} \alpha$. This situation, often referred to as 'Case C,' is discussed in detail by Xu et al. (1992). For the higher order Balmer lines, as well as the Paschen lines in the NIR, there is good agreement with the observations for this model. Similar agreement is found for the He I lines, where the comparatively high $\lambda 7065 / \lambda 5876$ ratio, as well as the $\lambda 10930 / \lambda 5876$ ratio, are a result of high optical depth in these lines (see Karamehmetoglu et al. 2019).

The Fe II emission shows a prominent line feature in the $\sim 8450$ $9200 \AA$ range, with the three peaks at 9076,9126 , and $9177 \AA$, which are well reproduced by both models. The lines at 8451 and $8490 \AA$ also agree well with the simulations, confirming the contribution of Ly $\alpha$ fluorescence. The feature at $\sim 2845 \AA$ is most likely to be Fe II rather than Mg I $\lambda \lambda 2852,2857$. This line complex consists of a number of strong lines from an upper $d^{4} D$ level fed by transitions from the $v^{4} F$ level, which also gives rise to the $\sim 9175 \AA$ peak. The simulations also show the very large number of Fe II lines in the region 4000-6000 $\AA$, which makes an unambiguous identification of other weak lines challenging.

The Sigut \& Pradhan (2003) model yields intensities that agree well at most wavelengths, although the $\sim 2848 \AA$ peak is overproduced by a factor of $\sim 3$. The $\eta$ Carinae spectrum gives better agreement with the optical range 4000-5500 $\AA$, while the AGN simulation agrees better with the fluorescence features in the NIR and UV. The relative line intensities depend on both the atomic data, especially collisional, and the physical conditions. The AGN environment, for example, has a density of $\sim 4 \times 10^{9} \mathrm{~cm}^{-3}$, which is much higher than that of the SN CSM. The calculated intensities also depend on the continuum level, which can be quite uncertain. Regardless of model, however, we find that there is strong evidence for the importance of $\mathrm{Ly} \alpha$ fluorescence in the spectrum of SN 2005ip.

\subsubsection{High-ionization CSM lines}

High-ionization ions were observed early (Smith et al. 2009b) and grew stronger with time (Smith et al. 2017). Fig. 4 shows that in our most recent spectra, we identify a large number of these highionization lines, including He II $\lambda 4685.6$, [O III] $\lambda \lambda 4363.2$, 4958.9, 5006.8, [Ne III] $\lambda \lambda 3869.1,3967.5$, [Ne V] $\lambda \lambda 3345.8,3425.5$, [Fe VII] $\lambda \lambda 3586.3,3758.9,4942.5,4988.6,5158.4,5720.7,6087.0,[\mathrm{Fe} \mathrm{X}]$ $\lambda 6374.5$, and [Fe XI] $\lambda$ 7891.8. In addition, [Ar X] $\lambda 5533$, [Ar XIV] $\lambda 4412.3$, and [Fe XIV] $\lambda 5302.9$ may be present, although likely blended with $[\mathrm{Fe} \mathrm{II}]$ lines at later epochs.

The numerous [Fe VII] lines offer a diagnostic of the density and temperature from the region where these arise. The FWHM of these lines is $\sim 350 \mathrm{~km} \mathrm{~s}^{-1}$, similar to the other high-ionization lines. The main uncertainty of the line fluxes comes from blending and the continuum level. The continuum uncertainty affects the $\lambda \lambda 4988.6$, 5158.4 lines most. The [Fe VII] $\lambda 6087.0$ line is an important diagnostic, but nearly coincides with the $\lambda 6086.37$ line of [Ca v]. From their transition rates, $[\mathrm{Ca} \mathrm{V}] \lambda 6086.37$ should have an intensity $\sim 0.19$ of $[\mathrm{Ca} \mathrm{V}] \lambda 5309.1$. The latter is a blend, but using its peak intensity as an upper limit to the flux and a ratio of the $\lambda 6086 / \lambda 5309$ features of 

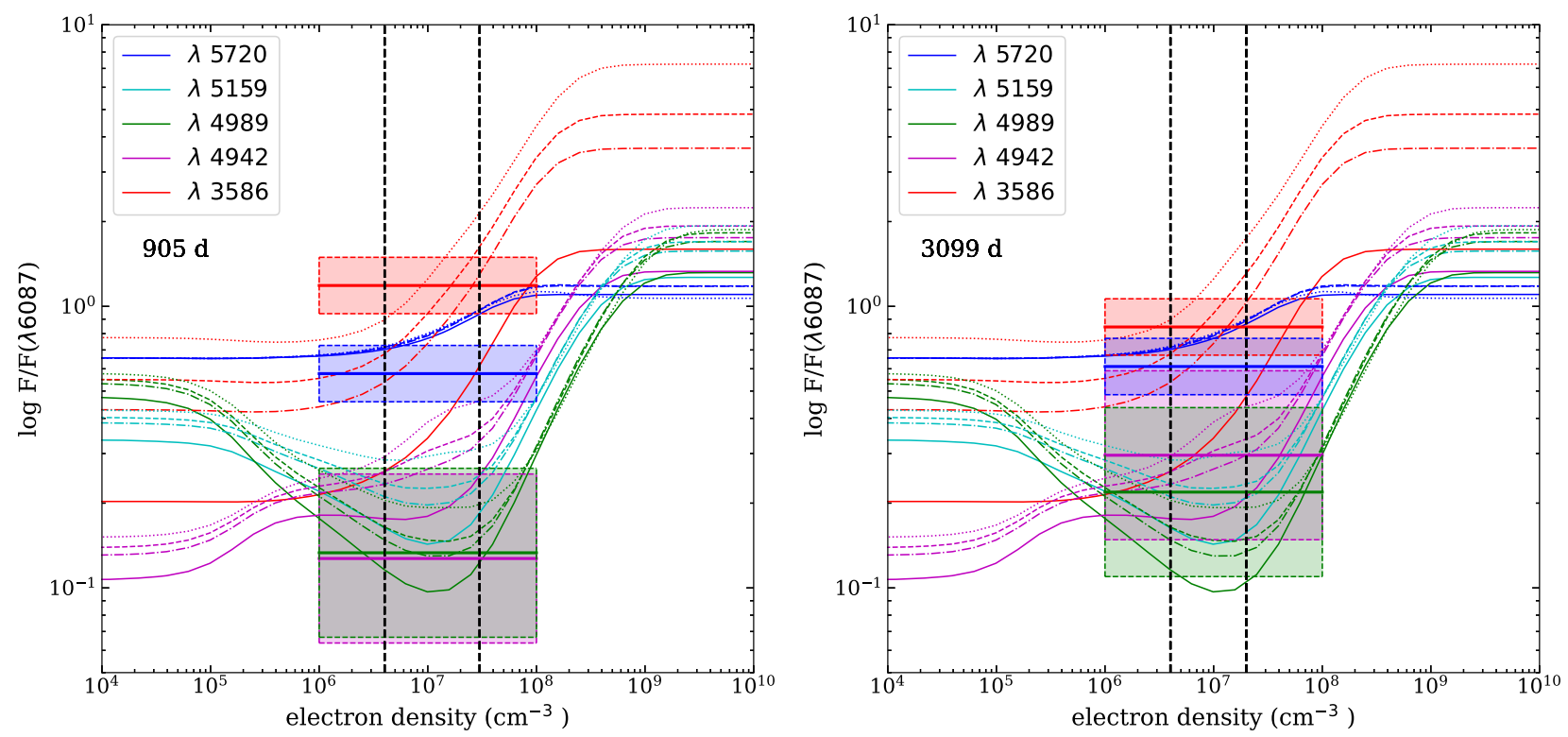

Figure 5. Line ratios of the optical [Fe VII] lines relative to the $\lambda 6086$ line as a function of density for $10000 \mathrm{~K}$ (solid lines), $20000 \mathrm{~K}$ (dotted-dashed lines), $30000 \mathrm{~K}$ (dashed), and $100000 \mathrm{~K}$ (dotted). The left-hand panel shows the observed ratios at $905 \mathrm{~d}$, while the right-hand panel is the same for $3099 \mathrm{~d}$. The vertical dashed lines shows the density range compatible with the different line ratios. The colour code of the modelled and observed ratios is given in the legend. The solid horizontal lines give the observed ratios, while the dashed lines indicate approximate error bars.

$\sim 2.4$, we predict a maximum contribution from $[\mathrm{Ca} \mathrm{V}]$ to the $\lambda 6086$ line of 8 per cent. We therefore conclude that this line is mainly due to [Fe VII]. The [Fe VII] $\lambda \lambda 3586.3,3758.9$ lines have a common upper level and their ratio is therefore fixed by their transition probabilities to $1: 1.5$, which agrees with the observed ratio, and we therefore only discuss the $\lambda \lambda 3586.3$ line.

From the observed spectrum at $905 \mathrm{~d}, F(\lambda 3586) / F(\lambda 6087) \approx 1.08$ and $F(\lambda 5721) / F(\lambda 6087) \approx 0.57$. At 3399,d the corresponding ratios are $F(\lambda 3586) / F(\lambda 6087) \approx 0.84$ and $F(\lambda 5721) / F(\lambda 6087) \approx 0.61$. The uncertainties in these ratios mainly come from the assumed reddening and the continuum level, and we estimate these to be $\sim 30$ per cent. Because of blending, the other ratios have considerably larger estimated uncertainties.

Fig. 5 plots the theoretical line ratios for the strongest lines as a function of the electron density for temperatures from $10^{4}$ to $10^{5} \mathrm{~K}$. Collision strengths are obtained from Berrington, Nakazaki \& Norrington (2000) and transition rates are obtained from NIST with a nine-level model atom. All ratios are relative to the strong $\lambda 6087.0$ line. Overplotted are the measured line ratios from days 905 and 3099.

We place the most emphasis on the strongest lines, represented by the $F(\lambda 3586) / F(\lambda 6087)$ and $F(\lambda 5721) / F(\lambda 6087)$ ratios. The strong temperature dependence of the $F(\lambda 3586) / F(\lambda 6087)$ ratio, together with the $F(\lambda 5721) / F(\lambda 6087)$ ratio, points to a temperature $\gtrsim 3 \times 10^{4} \mathrm{~K}$ on day 905 and $\gtrsim 2 \times 10^{4} \mathrm{~K}$ on day 3099 . Day 905 may have a temperature as high as $\sim 10^{5} \mathrm{~K}$. Photoionization calculations for X-ray-illuminated plasmas by Kallman \& McCray (1982, e.g. their Model 3) show that Fe VII is most abundant at $\sim 3 \times 10^{4} \mathrm{~K}$, consistent with these observations. For comparison, Fe XIV, which is seen in at least the earlier spectra, arises at $\sim 6 \times 10^{4} \mathrm{~K}$.

The electron density is $\sim 4 \times 10^{6}-3 \times 10^{7} \mathrm{~cm}^{-3}$ for day 905 , and a similar density range, $\sim 4 \times 10^{6}-2 \times 10^{7} \mathrm{~cm}^{-3}$, for day 3099 . It is difficult to draw any strong conclusions about a changing density between these epochs, but a decrease should have been expected. As pointed out by Berrington et al. (2000), there is likely considerable uncertainty in the atomic data, adding to the uncertainty in the observed fluxes. However, even considering this, it is quite remarkable that the $\mathrm{SN}$ is interacting with a CSM with density $\gtrsim 4 \times 10^{6} \mathrm{~cm}^{-3}$ at $\gtrsim 3000 \mathrm{~d}$ after explosion.

The density we find here can be compared to the density inferred from the bolometric light curve in Section 3.1. With a mass-loss rate $\dot{M} \approx 1 \times 10^{-2} \epsilon^{-1} \mathrm{M}_{\odot} \mathrm{yr}^{-1}$ and velocity $\sim 3000 \mathrm{~km} \mathrm{~s}^{-1}$ at $\sim 1000 \mathrm{~d}$, one obtains a density $\sim 4.5 \times 10^{6} \epsilon^{-1} \mathrm{~cm}^{-3}$ at $1000 \mathrm{~d}$. While this is compatible with the range we find above from the forbidden lines, one should note that the density from the bolometric light curve depends on $\epsilon$ and is therefore only a lower limit; it may be up to an order of magnitude higher. We also note that the forbidden lines are not affected by electron scattering and should therefore arise in a region different from that responsible for the bulk of the radiation, close to the shock. A natural scenario is therefore that the forbidden, high-ionization lines arise outside the region close to the shock which is optically thick to electron scattering, and where the Balmer lines and most of the radiation originate.

\subsubsection{The UV spectrum}

Fig. 6 shows the extracted UV spectra (no background subtraction is applied) and identifies the UV spectral lines, including the strong presence of $\mathrm{Ly} \alpha, \mathrm{NV} \lambda \lambda 1238.8,1242.8$, N IV $\lambda \lambda 1483.3$, 1486.5, N III] $\lambda \lambda 1746.8-1754.0, \mathrm{C}$ III] $\lambda \lambda 1906.7,1908.7$, and $\mathrm{Mg}$ II $\lambda \lambda 2795.5,2802.7$. Weaker lines also exist, specifically He II $\lambda 1640$, $\mathrm{N}$ II] $\lambda \lambda 2139.0,2142.8$, and $\mathrm{C}$ II] $\lambda \lambda 2323.5-2328.1$. We identify the feature at $\sim 2600 \AA$ with the Fe II $4 s a^{6} D$ to $4 p z^{6} D^{0}$ multiplet. There may be additional blends at $\sim 2500$ and $\sim 2750 \AA$, but their signal-tonoise ratio $(\mathrm{S} / \mathrm{N})$ is lower. We note that the $\mathrm{N}$ III] $\lambda \lambda 1746.8-1754.0$ multiplet is redshifted to $\sim 1758 \AA$.

Table 8 lists the reddening-corrected fluxes for the strongest UV lines from day 3065 shown in Fig. 6 (with the background level subtracted). Several caveats should be noted. The low $\mathrm{S} / \mathrm{N}$ in the region above $1700 \AA$ makes the continuum difficult to estimate, so the systematic error is approximated by comparing different wavelength ranges from the lines. The flux of C IV $\lambda \lambda 1548,1551$ 


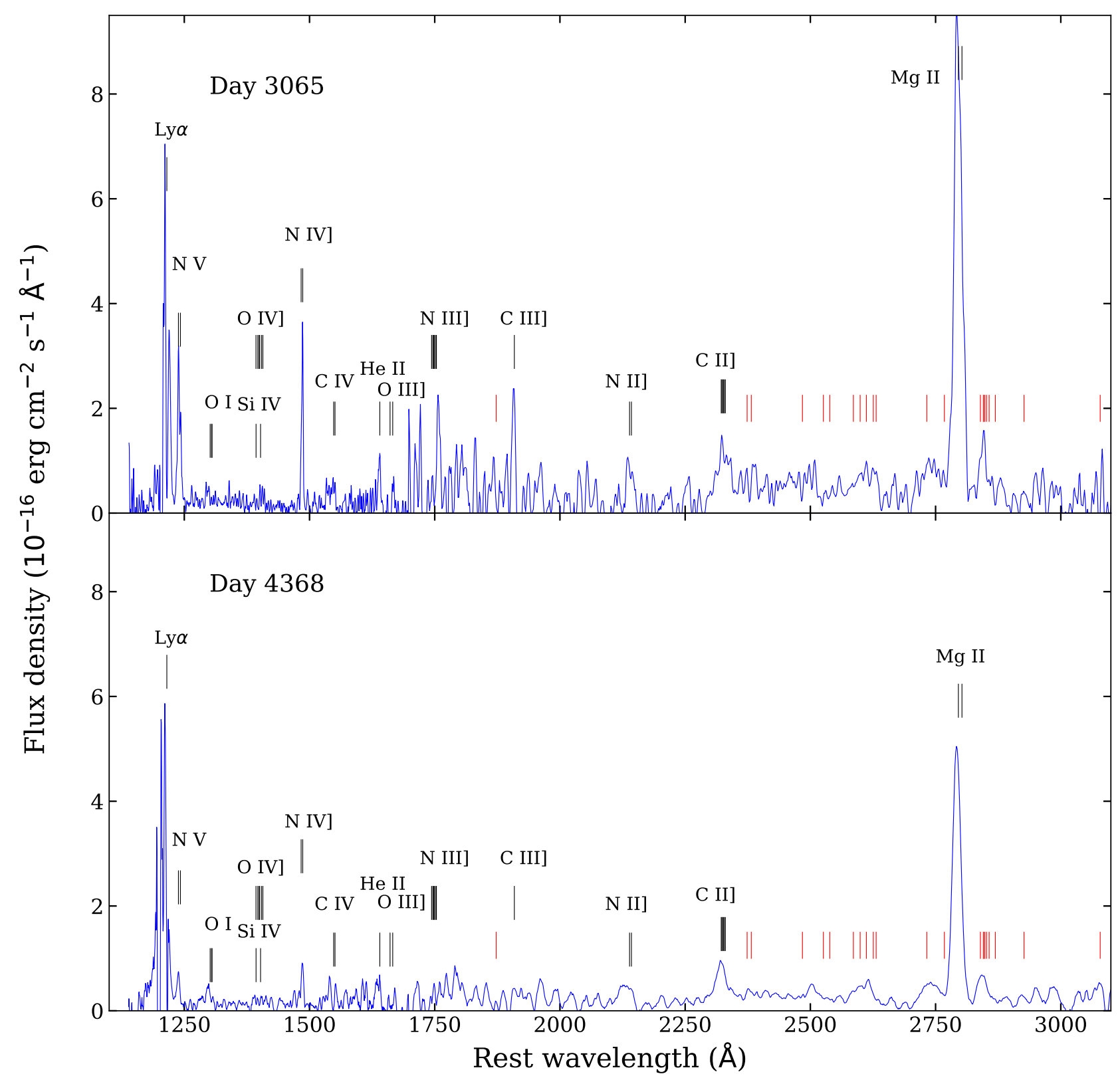

Figure 6. HST/STIS spectra of SN 2005ip on days 3065 and 4368 post-explosion. No extinction correction or background subtraction is applied. The wavelengths of probable line identifications are shown. The red vertical bars mark the wavelengths of the strongest Fe II lines from the list of Sigut \& Pradhan (2003).

Table 8. Reddening-corrected fluxes of strong UV lines from day 3065.

\begin{tabular}{llcc}
\hline Species & $\begin{array}{c}\text { Wavelength } \\
(\AA)\end{array}$ & $\begin{array}{c}\text { Flux } \\
\left(10^{-15} \mathrm{erg} \mathrm{s}^{-1} \mathrm{~cm}^{-2}\right)\end{array}$ \\
\hline $\mathrm{N} \mathrm{V}$ & $1238.8,1242.8$ & 4.80 & 0.07 \\
$\mathrm{~N} \mathrm{IV]}$ & $1483.3,1486.5$ & 2.72 & 0.15 \\
$\mathrm{C}_{\text {IV }}$ & $1548.2,1550.7$ & 1.14 & 0.11 \\
$\mathrm{He}$ II & 1640.4 & 0.88 & 0.26 \\
$\mathrm{O}$ III] & $1660.8,1666.2$ & $<0.5$ & - \\
$\mathrm{N}$ III] & $1746.8-1754.0$ & 3.01 & 0.35 \\
$\left.\mathrm{C}_{\text {III }}\right]$ & $1906.7,1908.7$ & 2.55 & 0.34 \\
\hline
\end{tabular}

is likely underestimated owing to the presence of both the Galactic and host-galaxy ISM absorption. The $\mathrm{O}$ III] $\lambda 1664$ line is weak and in a noisy region of the spectrum, so the calculated flux should only be considered an upper limit.

An estimate of the CSM density for the first epoch can be obtained from the ratio of the NIV] $\lambda 1483.3$ and $\lambda 1486.5$ lines, which we calculate to be $\lambda 1483.3 / \lambda 1486.5 \approx 0.3$. For an $X$-ray-ionized plasma, the N IV abundance peaks where the temperature is $(2-3) \times 10^{4} \mathrm{~K}$ (Kallman \& McCray 1982). Most important, the line ratio is sensitive to density but relatively insensitive to temperature. From fig. 1 of Keenan et al. (1995), the observed $\lambda 1483.3 / \lambda 1486.5$ ratio corresponds to an electron density of (2.0-3.6) $\times 10^{5} \mathrm{~cm}^{-3}$ in the temperature range $(1-2) \times 10^{4} \mathrm{~K}$. We conclude that the CSM density should be safely lower than the critical densities of the semiforbidden lines. 


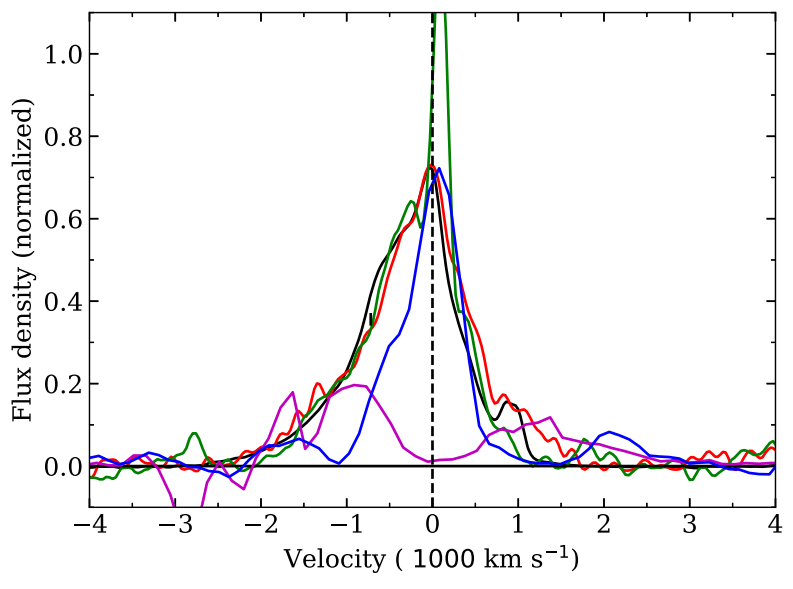

Figure 7. Line profiles of $\mathrm{H} \alpha$ (black), $\mathrm{H} \beta$ (green), He I $\lambda 5876$ (red), Ly $\alpha$ (magenta), and N IV] $\lambda \lambda 1483.3,1486.5$ (blue). Note the narrower line profile of the N IV] lines, as well as the contribution of the semiforbidden $\lambda 1483.3$ component on the blue side.

\subsubsection{Line profiles}

Fig. 7 compares the velocity profile of several UV and optical lines at $\sim 3100 \mathrm{~d}$. The $\mathrm{H} \alpha$ line extends to $\sim 2600 \mathrm{~km} \mathrm{~s}^{-1}$ on the blue side and only $\sim 1000 \mathrm{~km} \mathrm{~s}^{-1}$ on the red. $\mathrm{H} \beta, \mathrm{He}$ I $\lambda 5876$, and $\mathrm{Mg}$ II $\lambda \lambda 2796$, 2803 have similar line profiles. While the blue side can be well fitted with an electron-scattering wing, the line centroid is shifted to the blue, with a 'shoulder' at $\sim-800 \mathrm{~km} \mathrm{~s}^{-1}$. As shown by Taddia et al. (2020) and Dessart et al. (2015), this type of profile can be explained by a combination of emission from the shock wave and emission from the pre-shock CSM. The emission from the shock is responsible for the blueshifted shoulder, while the central component is coming from pre-ionized gas in the CSM. Both components are affected by electron scattering in the low-velocity CSM. This gives rise to the smooth, blue wing shortward of the 'shoulder.' Because of the smoothing by the electron scattering, the velocity of the 'shoulder' is lower than the shock velocity.

Both $\mathrm{H} \beta$ and $\mathrm{He}$ I $\lambda 5876$ have line profiles similar to that of $\mathrm{H} \alpha$, as expected if these arise from similar regions (Fig. 7). The Ly $\alpha$ line has a profile broadly consistent with $\mathrm{H} \alpha$, although strongly distorted by a central absorption feature from both the Galactic and host-galaxy ISM.

The C III], N III], N IV], and N V UV lines are considerably narrower than $\mathrm{H} \alpha$, and consistent with being unresolved at the $\sim 300 \mathrm{~km} \mathrm{~s}^{-1}$ instrumental resolution of STIS. These lines do not show the velocity shift of the Balmer and He I lines, and most likely come from the same optically thin, highly ionized region of the CSM as the highionization optical lines.

\subsubsection{Spectral evolution}

The optical spectra in this paper span one of the longest periods of any SN. Fig. 8 highlights only a very slow evolution of the spectrum over three epochs: 736, 3099, and $5105 \mathrm{~d}$. Unlike most other $\mathrm{SNe}$, there is no obvious transition to a nebular spectrum even at this extremely late stage. However, the line ratios do exhibit some interesting quantitative changes that are consistent with the shock wave propagating into a medium of decreasing density.

We start with a look at some with the most characteristic SN IIn lines: the $\mathrm{H} \alpha$ to $\mathrm{H} \beta$ ratio. Smith et al. (2017) showed this ratio was
10.8 at $109 \mathrm{~d}$ and 14.3 at $736 \mathrm{~d}$. It decreases from $\sim 24$ to $\sim 9.3$ to $\sim 5.4$ at days 905, 3099, and 5105, respectively. We note that the $\mathrm{H} \beta$ line is in a region of strong Fe II lines, which makes the background flux of this line difficult to estimate, especially for the spectrum at $905 \mathrm{~d}$. The $\mathrm{H} \alpha$ to $\mathrm{H} \beta$ ratio for this epoch is therefore especially uncertain. As discussed in Section 3.2.1, the general evolution of these line ratios indicates a decreasing optical depth in the Balmer lines.

The $\mathrm{H} \alpha$ blue wing has an exponential profile extending to $\sim 2700 \mathrm{~km} \mathrm{~s}^{-1}$ (see Fig. 9), indicating that electron scattering could still be important (Huang \& Chevalier 2018). The line has a visible deficit on the red side throughout the whole period, caused by either occultation from an optically thick photosphere or dust. The day 736 and $905 \mathrm{~d}$ spectra show a faint wing to higher velocity which is gradually fading away. Apart from this, the $\mathrm{H} \alpha$ line profile changes little over the period 905 to $5105 \mathrm{~d}$. Stritzinger et al. (2012) find a drop in the $\mathrm{H} \alpha$ flux by factor $\sim 10$ from 900 to $1800 \mathrm{~d}$, and as shown from the $r$-band photometry in Fig. 1, this trend has continued to our last observations.

The He I $\lambda 7065 / \lambda 5876$ ratio decreases from $\sim 0.56$ to $\sim 0.32$ from 905 to $5105 \mathrm{~d}$, again indicating a decrease in optical depth, similar to what we find for the Balmer lines. It is therefore most useful to compare individual line strengths to He I $\lambda 5876$, which is only moderately affected by optical-depth effects. The He I $\lambda 5876$ line has a very similar profile and evolution to $\mathrm{H} \alpha$. Both the [OI] $\lambda \lambda 6300,6364$ and the [CaII] $\lambda \lambda 7292,7324$ lines are relatively weak at $905 \mathrm{~d}$, but increase steadily relative to $\mathrm{H} \alpha$ over this period. This trend is again likely a result of decreasing electron density and, in turn, decreasing the effects of collisional deexcitaion. The width of the [O I] $\lambda 6300$ line is similar to $\mathrm{H} \alpha$ at these epochs. The $\mathrm{Mg}$ I] $\lambda 4571$ line, commonly seen in nebular spectra, is not detected above the noise, which is probably a result of the high UV flux above $7.65 \mathrm{eV}$, the ionization threshold of $\mathrm{Mg}$ I (see Fig. 6).

All of the high-ionization lines decrease relative to the He I $\lambda 5876$ line. While the decrease is modest between 905 and $3099 \mathrm{~d}$, it is steeper between 3099 and 5105 d (see Fig. 9 for the [Fe X] $\lambda 6374.5$ line). Typically, the relative fluxes decrease by a factor of $\sim 2$ over this time period. By contrast, the ratios of the $[\mathrm{Fe} \mathrm{VII}]$ lines change little over time. At $173 \mathrm{~d} F(\lambda 3586) / F(\lambda 6087) \approx 0.94 \pm 0.32$ and $F(\lambda 5721) / F(\lambda 6087) \approx 0.58 \pm 0.11$ (Smith et al. $2009 \mathrm{~b}$ ), which are similar to the ratios at 905 and $3099 \mathrm{~d}$.

In the UV, Fig. 6 compares the day 3065 and 4368 spectra. The day 4368 spectrum exhibits the same lines as the day 3065 spectrum, although the decreasing flux has caused several of the weaker lines to fade below the noise (Fig. 6). The weakest lines include in particular O III] $\lambda 1664, \mathrm{~N}$ III] $\lambda \lambda 1746.8-1754.0$, and C III] $\lambda \lambda 1906.7$, 1908.7, and a meaningful estimate of the $\mathrm{CNO}$ abundances can therefore not be made for this later epoch. The N IV] $\lambda \lambda 1483.3,1486.5$ lines are, however, still detected with a factor of $\sim 3.0$ lower flux compared to day 3065. Also, the $\mathrm{N} v \lambda \lambda 1238.8,1242.8$ doublet can be seen with a factor of $\sim 6.7$ lower flux. The strongest UV line is still the $\mathrm{Mg}$ II $\lambda \lambda 2795.5,2802.7$ doublet, which has decreased by a smaller factor, $\sim 1.7$.

The lines with the highest ionization stage (i.e. NV $\lambda \lambda 1238.8$, 1242.8) decrease fastest, followed by N IV] $\lambda \lambda 1483.3,1486.5$. This trend again illustrates that the general state of ionization in the lower density CSM has decreased considerably between the two epochs. This is consistent with the steadily decreasing X-ray luminosity (Fig. 1). Many of the higher ionization lines also disappeared or weakened around this same epoch in SN 1988Z (Smith et al. 2017). 


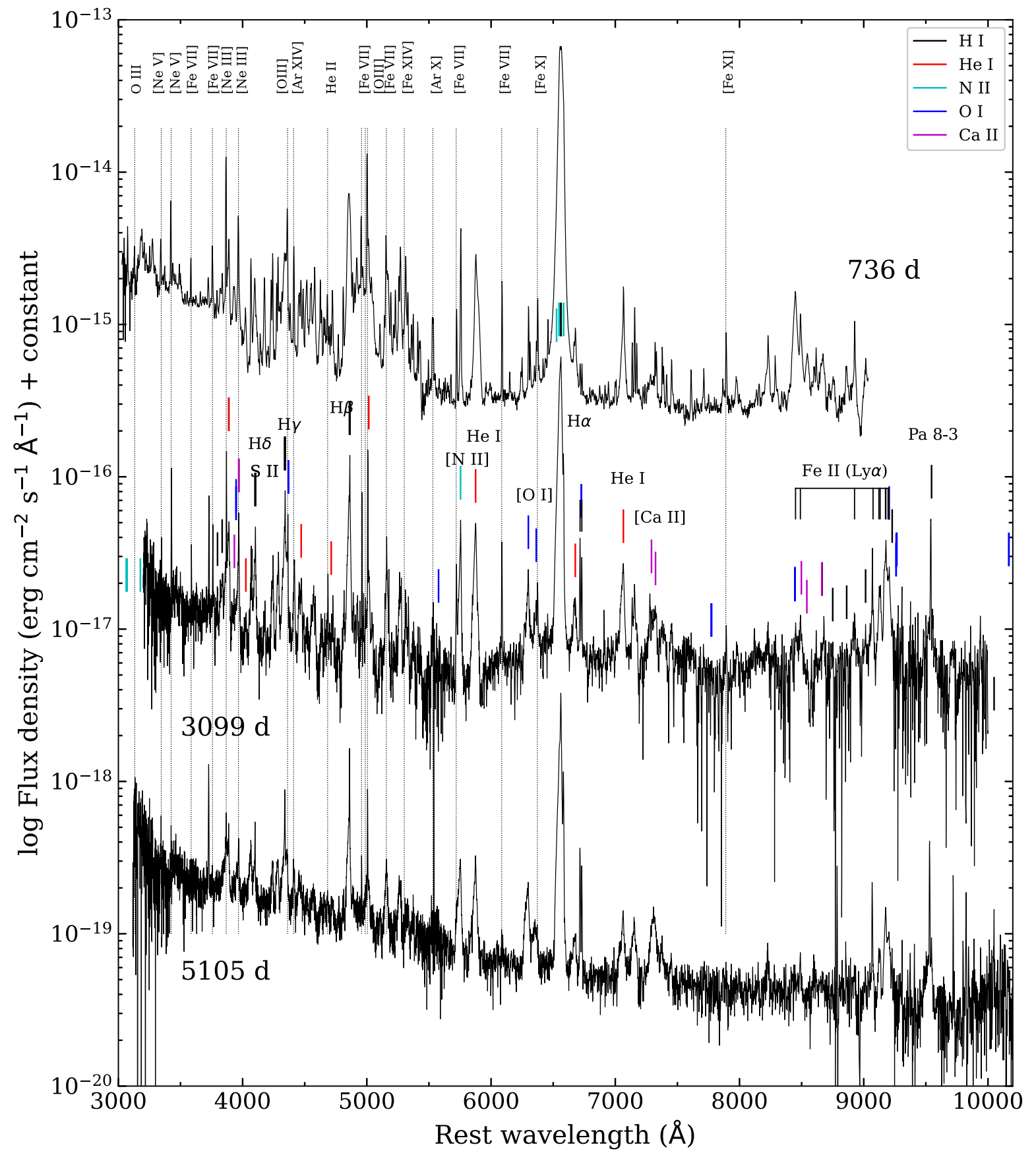

Figure 8. Evolution of the optical spectrum from day 736 to 5105 with identification of the high-ionization lines at the top and colour coding and identification of the low-ionization lines for the day 3099 spectrum.

\subsection{CNO processing abundances}

The relative abundances of ions listed in Table 8 depend on the temperatures and densities of the CSM. These lines have similar excitation energies, however, so the temperature sensitivity is relatively weak. If the density is less than the critical densities of these semiforbidden lines $\left(\sim 10^{9} \mathrm{~cm}^{-3}\right)$, then the density sensitivity is also weak.

On day $\sim 100$, Smith et al. (2009b) use the critical densities of the high-ionization optical coronal lines to set an upper limit on the density of the pre-shocked CSM at $<10^{7}-10^{8} \mathrm{~cm}^{-3}$. The absence of the [O II] $\lambda \lambda 3726,3729$ doublet before day 173 sets a lower limit on the density of $\gtrsim 3 \times 10^{5} \mathrm{~cm}^{-3}$. However, the [S II] $\lambda \lambda 6717$,
6731 doublet ratio on day 173 yields a density of only $\sim 10^{2} \mathrm{~cm}^{-3}$ Smith et al. (2009b) suggest that this inconsistency may indicate an inhomogeneous CSM. These measurements correspond to days 109-173, so we may infer the densities by day 3000 could be lower by a factor of $\sim(3000 / 100)^{2} \approx 10^{2}$ for a steady wind. However, a steady wind may not apply for a time-limited eruption and clumpiness further complicates this.

Using the above temperature and density information, we calculate the elemental abundances using the ionic flux ratios from Table 8 and atomic data from the CHIANTI compilation (Landi et al. 2012). The values given correspond to ratios using temperatures in the range $(1.0-3.0) \times 10^{4} \mathrm{~K}$ and densities up to $10^{6} \mathrm{~cm}^{-3}$. To calculate 


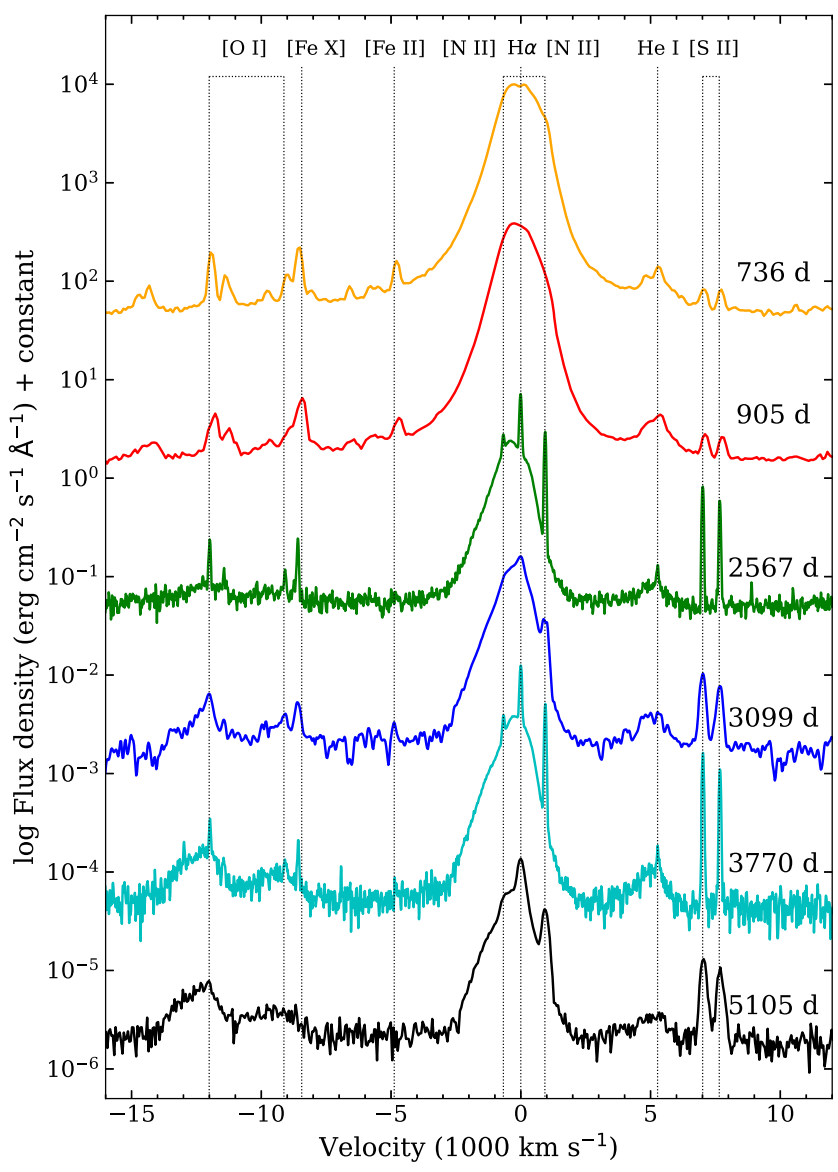

Figure 9. Evolution of the $\mathrm{H} \alpha$ and [O I] line profiles from day 736 to 5105 . The flux of each spectrum is normalized, put on a logarithmic scale, and shifted by a constant. Note the nearly exponential profile on the blue side of the lines at the last four epochs, characteristic of electron scattering. Also note the emergence of the broad [O I] lines at $\sim 3000 \mathrm{~d}$ and the fading of the $[\mathrm{Fe} x]$ line in the last spectrum. The smaller FWHM of the narrow lines in the day 2567 and 3770 spectra is caused by the higher spectral resolution in these data.

Table 9. Ionic abundance ratios.

\begin{tabular}{lccc}
\hline $\mathrm{N}_{\text {III }} / \mathrm{C}_{\text {III }}$ & $\mathrm{N}$ IV/C IV & $\mathrm{N} \mathrm{III/O} \mathrm{III}$ & N IV/O III \\
\hline $9.5-12.8 \pm 2.3$ & $15.7-17.1 \pm 2.0$ & $>3.3-6.0$ & $1.2-2.0$ \\
\hline
\end{tabular}

the elemental abundances, however, requires assumptions about the ionization structure of the CSM. X-ray photoionized models characterized by a $10 \mathrm{keV}$ bremsstrahlung spectrum reveal that the C III and N III zones, as well as the C IV, N IV, and O III zones, nearly coincide (Kallman \& McCray 1982). SN 2005ip has a somewhat different ionizing spectrum and a lower CSM density, so this model is not necessarily true. However, the presence of numerous highionization lines, like $[\operatorname{Ar} \mathrm{X}] \lambda 5536$, as well as the direct observations of a high X-ray luminosity, argue for X-ray ionization to dominate, although the exact spectrum is uncertain. Because more detailed photoionization models do not exist, we assume here a similar ionization structure as described by Kallman \& McCray (1982).

Table 9 lists the abundance ratios of C III-IV, N III-IV, and O III: $\mathrm{N} / \mathrm{C}=9.5-12.8 \pm 2.3$ and N/O $>1.2-2.0$. Since the C IV line is severely suppressed by ISM absorption from the host galaxy, we did not include the N IV/C IV ratio in the analysis. Owing to the closer correspondence of the $\mathrm{N}$ IV and $\mathrm{O}$ III zones in the models, we prefer to use these ions to calculate the N/O ratio. Independent of uncertainties in densities, temperatures, and ionization structure, these results show that the CSM of SN 2005ip is strongly N enriched. Compared to solar (Asplund et al. 2009) the above ratios are enhanced by a factors of $38-51$ for N/C and $>8.7-14$ for N/O, consistent with products from CNO burning.

\subsection{Grain heating and dust mass}

The Spitzer data can provide important constraints on the dust heating and total dust mass. The assumed physical scenario is that a dense, external dust shell was formed during a pre-SN eruption and is now heated by internal optical, UV, and X-ray emission generated by shock interaction (Section 3.1, Fox et al. 2011). We use the 3D radiative transfer code Monte Carlo Simulations of Ionized Nebulae (MOCCASIN, Ercolano, Barlow \& Storey 2005, and references within) to analyse data during the Spitzer epochs closest in time to the two epochs of HST/STIS UV spectroscopy (e.g. 2015 and 2018). MOCASSIN accepts multiple parameters defined by a user and executes the desired scenario on a Cartesian grid. Both epochs were initially modelled with a simple blackbody and then again with a modified blackbody. We assume a spherical shell with 100 per cent amorphous carbon dust distributed uniformly and a standard Mathis, Rumpl \& Nordsieck (1977, MRN) grain size distribution of $a^{-3.5}$ between 0.005 and $0.05 \mu \mathrm{m}$. The model accounts only for the observed dust, which is most likely the hottest dust at the inner radius of the external shell of material. A much higher mass (10-100 times more) of colder dust likely exists (e.g. Matsuura 2017).

The 2018 epoch was modelled first since it has accompanying ground-based optical photometry from the LBT and NIR photometry from UKIRT. The best-fitting model suggests a shell with $R_{\text {in }}=$ $9.0 \times 10^{15} \mathrm{~cm}$ and $R_{\text {out }}=6.0 \times 10^{16} \mathrm{~cm}$. The dust temperature ranges from 700 to $600 \mathrm{~K}$ at the inner and outer edges, respectively. The implied dust mass is $1.59 \times 10^{-3} \mathrm{M}_{\odot}$ with an equivalent optical depth of 2.46. The addition of the UV spectrum resulted in a minimal increase in the dust temperature $(\Delta T \approx 10 \mathrm{~K})$ and IR brightness $\left(\Delta F_{v} \approx 0.01 \mathrm{mJy}\right)$.

For the 2015 spectrum, we used the same smooth shell model derived for the 2018 fit and an input luminosity of $1.35 \times 10^{41} \mathrm{erg} \mathrm{s}^{-1}$ $\left(\sim 3.51 \times 10^{7} \mathrm{~L}_{\odot}\right)$. The temperature of the dust was found to range from about $720-530 \mathrm{~K}$ from the inner to outer radius of the shell, respectively. The implied dust mass and equivalent optical depth were also the same as in the 2018 model. The addition of the UV spectrum to the model resulted in slightly higher dust temperatures ( $\Delta T \approx 10-40 \mathrm{~K}$, by grain size) and IR brightness ( $\left.\Delta F_{v} \approx 0.1 \mathrm{mJy}\right)$.

\subsection{The dust powering mechanism}

Following a nearly $2000 \mathrm{~d}$ plateau, the MIR evolution qualitatively follows a similar slow decline as the optical of $\sim 1 \mathrm{mag}(1000 \mathrm{~d})^{-1}$, although there may be an indication of a slower decline at the latest epochs. The MIR Spitzer photometry from two earlier epochs (days 948 and 2057) are already shown to be consistent with a large, preexisting dust shell that is continuously heated by visible and X-ray radiation generated by ongoing CSM interaction (Fox et al. 2010, 2011). Assuming a spherically symmetric dust shell located at the blackbody radius, the MIR would require a visible/X-ray heating luminosity from CSM interaction of $\sim 10^{8} \mathrm{~L}_{\odot}$ at these epochs (see fig. 10b of Fox et al. 2011). This luminosity is consistent with the $r$-band photometry on day 948 given in fig. 10 of Smith et al. (2009b) 
or from fig. 9 of Stritzinger et al. (2012), based on the full optical through IR SED.

Another consistency check requires that the optical depth, $\tau$, for such a system be $>1$. Following equation (23) from Dwek et al. (2017), the dust's optical depth at wavelength $\lambda$ can be written as

$\tau(\lambda)=Z_{\mathrm{dH}} m_{H} \kappa_{\mathrm{ext}}(\lambda) N_{\mathrm{H}}$,

where $Z_{\mathrm{dH}}$ is the dust-to-H mass ratio, $m_{H}$ is the hydrogen atomic mass, $\kappa_{\text {ext }}$ is the extinction coefficient, and $N_{\mathrm{H}}$ is the column depth. The X-ray observations in both this paper and Katsuda et al. (2014) show a column depth, $N_{\mathrm{H}}$, that decreases from $\sim 5 \times 10^{22}$ to $0.1 \times 10^{22} \mathrm{~cm}^{-2}$ over time. From fig. 4 of Fox et al. (2010), we assume $10^{4}<\kappa_{\text {ext }}(V)<10^{6} \mathrm{~cm}^{-2} \mathrm{~g}$, which represents a typical value for a variety of grain sizes $<1.0 \mu \mathrm{m}$ and compositions. For these values, we derive $\tau(V) \approx 1.7 \times 10^{2} Z_{\mathrm{dH}}$. For typical gas-todust mass ratios of $\sim 100\left(Z_{\mathrm{dH}} \approx 0.01\right)$, this implies an optical depth $\tau>1$ throughout the latest epochs for most values of $\kappa$, typically corresponding to smaller grains. While these are order-of-magnitude approximations, a value $\tau>1$ for a spherical shell would impact the measured extinction and total radiated energy. The fact that both the measured extinction is low (Stritzinger et al. 2012) and the optical to mid-IR hovers around $\sim 1$ suggests that the geometry is likely more complicated than a spherical shell, perhaps clumpy or an anisotropic shell or disc (Smith et al. 2009b; Fox et al. 2009, 2010; Stritzinger et al. 2012; Smith et al. 2017).

\section{SUMMARY}

We have presented very late-time observations of SN 2005ip, including Chandra/ACIS X-ray spectra, HST/STIS UV spectra, HST/WFC3 optical photometry, ground-based optical and NIR photometry, and Spitzer/IRAC photometry. The MIR evolution is consistent with the previously proposed pre-existing dust shell that is radiatively heated by ongoing CSM interaction. There may be some indication of a relatively slower MIR decline in the latest epochs, which could suggest a slowly decaying thermal echo. The total energy radiated by the shock so far is in excess of $10^{50} \mathrm{erg}$. The large energy release indicates an efficient conversion of kinetic energy to radiation. The progenitor mass-loss rate we find is $\gtrsim 1 \times 10^{-2} \mathrm{M}_{\odot} \mathrm{yr}^{-1}$, and the total mass lost is $\gtrsim 1 \mathrm{M}_{\odot}$ but can be considerably larger, depending on the exact efficiency for the conversion of shock energy to radiation. This explosion is thought to arise from a massive, luminous red supergiant, like VY CMa (Smith, Hinkle \& Ryde 2009a), or an LBV progenitor in order to account for the huge mass-loss rate (Fox et al. 2009; Smith et al. 2009b; Stritzinger et al. 2012). The optical spectra show strong effects of Ly $\alpha$ fluorescence and a decreasing optical depth in the lines. The UV spectra show that the CSM of SN 2005ip is strongly $\mathrm{N}$ enriched, consistent with products from $\mathrm{CNO}$ burning.

After more than $5 \mathrm{yr}$ of a relatively flat plateau, the light curve has begun to fade in all bands. This result indicates that the shock may finally be reaching the outer extent of the dense CSM shell around SN 2005ip. The final optical and MIR photometry, however, leaves some ambiguity that the declining CSM interaction could still be continuing at a lesser strength, and only time will tell if the demise is permanent.

\section{ACKNOWLEDGEMENTS}

We thank the anonymous referee for suggestions that improved this paper. Some of the data presented herein were obtained at the W. M. Keck Observatory, which is operated as a scientific partnership among the California Institute of Technology, the University of
California, and the National Aeronautics and Space Administration (NASA); the Observatory was made possible by the generous financial support of the W. M. Keck Foundation. The authors wish to recognize and acknowledge the very significant cultural role and reverence that the summit of Maunakea has always had within the indigenous Hawaiian community; we are most fortunate to have the opportunity to conduct observations from this mountain. We thank WeiKang Zheng for helping obtain Keck optical photometry and spectroscopy. When the data reported here were acquired, the United Kingdom InfraRed Telescope (UKIRT) was supported by NASA and operated under an agreement among the University of Hawaii, the University of Arizona, and Lockheed Martin Advanced Technology Center; operations were enabled through the cooperation of the East Asian Observatory. The Large Binocular Telescope (LBT) is an international collaboration among institutions in the United States, Italy, and Germany. The LBT Corporation partners are The University of Arizona on behalf of the Arizona university system; Istituto Nazionale di Astrofisica, Italy; LBT Beteiligungsgesellschaft, Germany, representing the Max Planck Society, the Astrophysical Institute Potsdam, and Heidelberg University; The Ohio State University; The Research Corporation, on behalf of The University of Notre Dame, University of Minnesota and University of Virginia. Observations reported here were obtained at the Multiple Mirror Telescope (MMT) Observatory, a joint facility of the University of Arizona and the Smithsonian Institution. This publication makes use of data products from the 2MASS, which is a joint project of the University of Massachusetts and the Infrared Processing and Analysis Center/California Institute of Technology, funded by NASA and the U.S. National Science Foundation (NSF).

This work is based in part on observations obtained with the Spitzer Space Telescope, which is operated by the Jet Propulsion Laboratory, California Institute of Technology, under a contract with NASA. Financial support for this work was provided by NASA through grants GO-10877, GO-13287, GO-14598, GO-14688, and GO-15166 from the STScI, which is operated by the Associated Universities for Research in Astronomy, Inc. (AURA), under NASA contract NAS 5-26555. AVF's supernova group has also been supported by NASA/Chandra grant GO7-18067X, the Christopher R. Redlich Fund, the TABASGO Foundation, NSF grant AST1211916, and the Miller Institute for Basic Research in Science (U.C. Berkeley). CF acknowledges support from the Swedish Research Council and Swedish National Space Board. MDS is supported by generous grants (13261 and 28021) from Villum Fonden and by a project grant (8021-00170B) from the Independent Research Fund Denmark. TS is supported by the GINOP-2-3-2-15-201600033 project ('Transient Astrophysical Objects') of the National Research, Development and Innovation Office (NKFIH), Hungary, funded by the European Union.

\section{DATA AVAILABILITY}

The data underlying this article will be shared on reasonable request to the corresponding author.

\section{REFERENCES}

Ahn C. P. et al., 2012, ApJS, 203, 21

Anderson H., Ballance C. P., Badnell N. R., Summers H. P., 2002, J. Phys B: At. Mol. Phys., 35, 1613

Asplund M., Grevesse N., Sauval A. J., Scott P., 2009, ARA\&A, 47, 481

Benjamin R. A., Skillman E. D., Smits D. P., 1999, ApJ, 514, 307

Berrington K. A., Nakazaki S., Norrington P. H., 2000, A\&AS, 142, 313 
Bevan A. et al., 2018, MNRAS, 485, 5192

Blackburn J. K., 1995, in Shaw R. A., Payne H. E., Hayes J. J. E., eds, ASP Conf. Ser., Vol. 77, Astronomical Data Analysis Software and Systems IV. Astron. Soc. Pac., San Francisco, p. 367

Boles T., Nakano S., Itagaki K., 2005, Central Bureau Electronic Telegrams, 275,1

Butler N. et al., 2012, Proc. SPIE, 8446, 10

Cardelli J. A., Clayton G. C., Mathis J. S., 1989, ApJ, 345, 245

Chevalier R. A., Fransson C., 1994, ApJ, 420, 268

Chevalier R. A., Fransson C., 2017, in Alsabti A. W., Murdin P., eds, Thermal and Non-thermal Emission from Circumstellar Interaction, Handbook of Supernovae. p. 875

Chevalier R. A., Irwin C. M., 2011, ApJ, 729, L6

Contreras C. et al., 2010, AJ, 139, 519

Dessart L., Audit E., Hillier D. J., 2015, MNRAS, 449, 4304

Dolphin A., 2016, Astrophysics Source Code Library, record ascl:1608.013

Dwek E. et al., 2017, ApJ, 847, 91

Ercolano B., Barlow M. J., Storey P. J., 2005, MNRAS, 362, 1038

Faber S. M. et al., 2003, Proc. SPIE, 4841, 1657

Fazio G. G. et al., 2004, ApJS, 154, 10

Filippenko A. V., 1982, PASP, 94, 715

Filippenko A. V., 1997, ARA\&A, 35, 309

Foley R. J. et al., 2003, PASP, 115, 1220

Fox O. D. et al., 2009, ApJ, 691, 650

Fox O. D., Chevalier R. A., Dwek E., Skrutskie M. F., Sugerman B. E. K., Leisenring J. M., 2010, ApJ, 725, 1768

Fox O. D. et al., 2011, ApJ, 741, 7

Fox O. D. et al., 2012, Proc. SPIE, 8453, 59

Fox O. D., Filippenko A. V., Skrutskie M. F., Silverman J. M., Ganeshalingam M., Cenko S. B., Clubb K. I., 2013, AJ, 146, 2

Fransson C. et al., 2002, ApJ, 572, 350

Fransson C. et al., 2005, ApJ, 622, 991

Fransson C. et al., 2014, ApJ, 797, 118

Garmire G. P., Bautz M. W., Ford P. G., Nousek J. A., Ricker G. R. Jr, 2003, in Truemper J. E., Tananbaum H. D., eds, Proc. SPIE Conf. Ser., Vol. 4851, X-Ray and Gamma-Ray Telescopes and Instruments for Astronomy. SPIE, Bellingham, p. 28

Habergham S. M., James P. A., Anderson J. P., 2012, MNRAS, 424, 2841

Hamuy M. et al., 2006, PASP, 118, 2

Hartman H., 2013, Fluorescence in Astrophysical Plasmas. p. 189

Horne K., 1986, PASP, 98, 609

Houck J. R. et al., 2004, ApJS, 154, 18

Huang C., Chevalier R. A., 2018, MNRAS, 475, 1261

Johansson S., Hamann F. W., 1993, Phys. Scr. Vol. T, 47, 157

Johansson S., Jordan C., 1984, MNRAS, 210, 239

Kallman T. R., McCray R., 1982, ApJS, 50, 263

Karamehmetoglu E. et al., 2019, preprint (arXiv:1910.06016)

Katsuda S., Maeda K., Nozawa T., Pooley D., Immler S., 2014, ApJ, 780, 184

Keenan F. P., Ramsbottom C. A., Bell K. L., Berrington K. A., Hibbert A., Feibelman W. A., Blair W. P., 1995, ApJ, 438, 500

Landi E., Zanna G. D., Young P. R., Dere K. P., Mason H. E., 2012, ApJ, 744, 99

Matheson T., Filippenko A. V., Ho L. C., Barth A. J., Leonard D. C., 2000, AJ, 120, 1499

Mathis J. S., Rumpl W., Nordsieck K. H., 1977, ApJ, 217, 425

Matsuura M., 2017, Dust and Molecular Formation in Supernovae. p. 2125

Mauerhan J. C. et al., 2013, MNRAS, 430, 1801

Moriya T. J., Maeda K., Taddia F., Sollerman J., Blinnikov S. I., Sorokina E. I., 2013, MNRAS, 435, 1520

Nielsen A.-S. B., Hjorth J., Gall C., 2018, A\&A, 611, A67

Nomoto K. I., Iwamoto K., Suzuki T., 1995, Phys. Rep., 256, 173

Ofek E. O. et al., 2014, ApJ, 781, 42

Oke J. B. et al., 1995, PASP, 107, 375

Schlegel E. M., 1990, MNRAS, 244, 269

Sigut T. A. A., Pradhan A. K., 1998, ApJ, 499, L139

Sigut T. A. A., Pradhan A. K., 2003, ApJS, 145, 15
Silverman J. M. et al., 2012, MNRAS, 425, 1789

Skrutskie M. F. et al., 2006, AJ, 131, 1163

Smith N., 2014, ARA\&A, 52, 487

Smith N., 2017, Interacting Supernovae: Types IIn and Ibn. p. 403

Smith N., Tombleson R., 2015, MNRAS, 447, 598

Smith N. et al., 2007, ApJ, 666, 1116

Smith N., Chornock R., Li W., Ganeshalingam M., Silverman J. M., Foley R. J., Filippenko A. V., Barth A. J., 2008, ApJ, 686, 467

Smith N., Hinkle K. H., Ryde N., 2009a, AJ, 137, 3558

Smith N. et al., 2009b, ApJ, 695, 1334

Smith N. et al., 2010, AJ, 139, 1451

Smith N., Li W., Silverman J. M., Ganeshalingam M., Filippenko A. V., 2011, MNRAS, 415, 773

Smith N. et al., 2017, MNRAS, 466, 3021

Stritzinger M. et al., 2012, ApJ, 756, 173

Szalai T., Zsíros S., Fox O. D., Pejcha O., Müller T., 2019, ApJS, 241, 38

Taddia F. et al., 2015, A\&A, 580, A131

Taddia F. et al., 2020, A\&A, 638, A92

Wade R. A., Horne K., 1988, ApJ, 324, 411

Watson A. M. et al., 2012, Proc. SPIE Vol. 8444, Ground-based and Airborne Telescopes IV. SPIE, Bellingham, p. 8444

Williams B. J., Fox O. D., 2015, ApJ, 808, L22

Xu Y., McCray R., Oliva E., Rand ich S., 1992, ApJ, 386, 181

Zethson T., Johansson S., Hartman H., Gull T. R., 2012, A\&A, 540, A133

${ }^{1}$ Space Telescope Science Institute, 3700 San Martin Drive, Baltimore, MD 21218, USA

${ }^{2}$ Department of Astronomy, Oskar Klein Centre, Stockholm University, AlbaNova, SE-106 91 Stockholm, Sweden

${ }^{3}$ Steward Observatory, 933 N. Cherry Ave., Tucson, AZ 85721, USA

${ }^{4}$ Department of Physics, University of California, Davis, CA 95616, USA

${ }^{5}$ Department of Astronomy, University of California, Berkeley, CA 94720-

3411, USA

${ }^{6}$ NASA Goddard Space Flight Center, 8800 Greenbelt Road, Greenbelt, DMD 20771, USA

${ }^{7}$ Joint Space-Science Institute, University of Maryland, College Park, MD 20742, USA

${ }^{8}$ Department of Physics and Astronomy, Louisiana State University, Baton Rouge, LA 70803, USA

${ }^{9}$ Miller Institute for Basic Research in Science, University of California, Berkeley, CA 94720, USA

${ }^{10}$ Center for Interdisciplinary Exploration and Research in Astrophysics (CIERA) and Department of Physics and Astronomy, Northwestern University, Evanston, IL 60208, USA

${ }^{11}$ University of Cincinnati Blue Ash College, 9555 Plainfield Rd., Blue Ash, $\mathrm{OH} 45236$, USA

${ }^{12}$ Minnesota Institute for Astrophysics, University of Minnesota, 115 Union St. SE, Minneapolis, MN 55455, USA

${ }^{13}$ Department of Astronomy and Astrophysics, University of California, Santa Cruz, CA 95064, USA

${ }^{14}$ The Aerospace Corporation, 2310 E. El Segundo Blvd., El Segundo, CA 90245, USA

${ }^{15}$ Jet Propulsion Laboratory, 4800 Oak Grove Drive, MS 169-506, Pasadena, CA 91109, USA

${ }^{16}$ California Institute of Technology, Pasadena, CA 91125, USA

${ }^{17}$ SOFIA-USRA, NASA Ames Research Center, Mail Stop N232-12, Moffett Field, CA 94035-1000, USA

${ }^{18}$ Department of Physics and Astronomy, Aarhus University, Ny Munkegade 120, DK-8000 Aarhus C, Denmark

${ }^{19}$ Department of Optics and Quantum Electronics, University of Szeged, $\mathrm{H}$ 6720 Szeged, Dóm tér 9., Hungary

${ }^{20}$ Konkoly Observatory, Research Centre for Astronomy and Earth Sciences, H-1121 Budapest, Konkoly Thege Miklós út 15-17, Hungary

${ }^{21}$ IPAC/Caltech, Mailcode 100-22, Pasadena, CA 91125, USA

This paper has been typeset from a $\mathrm{T}_{\mathrm{E}} \mathrm{X} / \mathrm{LAT} \mathrm{E}$ file prepared by the author. 